\title{
BIRDS
}

\section{3rd ANNUAL SASKATCHEWAN CHRISTMAS BIRD COUNT-2004}

\author{
ALAN R. SMITH, 115 Perimeter Road, Saskatoon SK S7N 0X4 and ROBERT E. \\ JOHANSON, $4061255^{\text {th }}$ Avenue N, Saskatoon SK S7K 6A5
}

\section{The Counts}

Although the dates of the Christmas Bird Count have been fixed at 14 December to 5 January since the winter of 2000-2001, few compilers have taken advantage of the days at the beginning of the count period to conduct counts. This year was no exception as only 15 counts were conducted on or before the weekend of December 17-18. That weekend was the last hurrah for the fall-like weather. By Monday, December 19, true winter weather arrived with a vengeance. As you can imagine, a change for the worse in the weather can have a measurable influence on the number of bird species seen; counts taken on or before December $18^{\text {th }}$ averaged 20 species, those after that date averaged 18 species.

Four new counts were established this year. They are Cherry Lake (south of Indian Head), Churchbridge, McNutt and Morse. After absences of two or more years, we welcome the return of counts conducted at Grenfell, Kyle, Little Manitou Lake and Moose Jaw. All in all, 607 observers in 99 localities participated in the Christmas Bird Count this winter.

Two counts, Indian Head and Kamsack, included in this year's tabulations do not represent one day counts but consist of observations made over a period of days during the count period. This information is valuable and should be submitted, but because it is not comparable to the results of the other counts, which are from one day only, it will not, in future, be tabulated with the other results. The same applies to count results from more than one count location or observations made outside the count period.

\section{The birds}

Just over 155,000 birds of 103 species were recorded on count day. An additional three species were recorded during the count period. For the third consecutive year Saskatoon led all counts with 46 species on count day plus another four during the count period. This ties their previous count day total, but exceeds the count period record by one species.

\section{Population trends}

Canada Goose and Mallard numbers were up, due mainly to the return of the Coronach count (Table 7). Upland game birds returned to earth from their unprecedented numbers last year. Diurnal raptor numbers were stable except that no Gyrfalcons were identified with certainty for the first time since 1986 . Most owls showed increases, with record high counts set for both Great Horned and Short-eared Owls at Kyle.

The news was mixed for boreal forest passerines with Northern Shrikes and Brown Creepers way up, while Gray Jays and Boreal Chickadees were way down. Open country passerines, Horned Larks, Snow Buntings and Lapland Longspurs, were all up over last year. Although still seen in small numbers, record high counts were set or tied for all three of our common species of Zonotrichia sparrow (Table 6). 


\section{New Species}

Last year we added two species that we did not expect: a Sandhill Crane at Yorkton and a Common Crane at Leader. This year we added two species more likely to be found. The first is the Cackling Goose, which has recently been elevated to species status from the larger Canada Goose, so it was only a matter of time before this species was recorded on a CBC. Only one was seen, however, and it was on the Gardiner Dam count. The other species has long been expected, at least in the Cypress Hills, and this is the American Dipper seen on the Eastend count. There are eleven previous records including one winter record for the species in the province. These two new species bring the all-time Saskatchewan CBC list to 179 species.

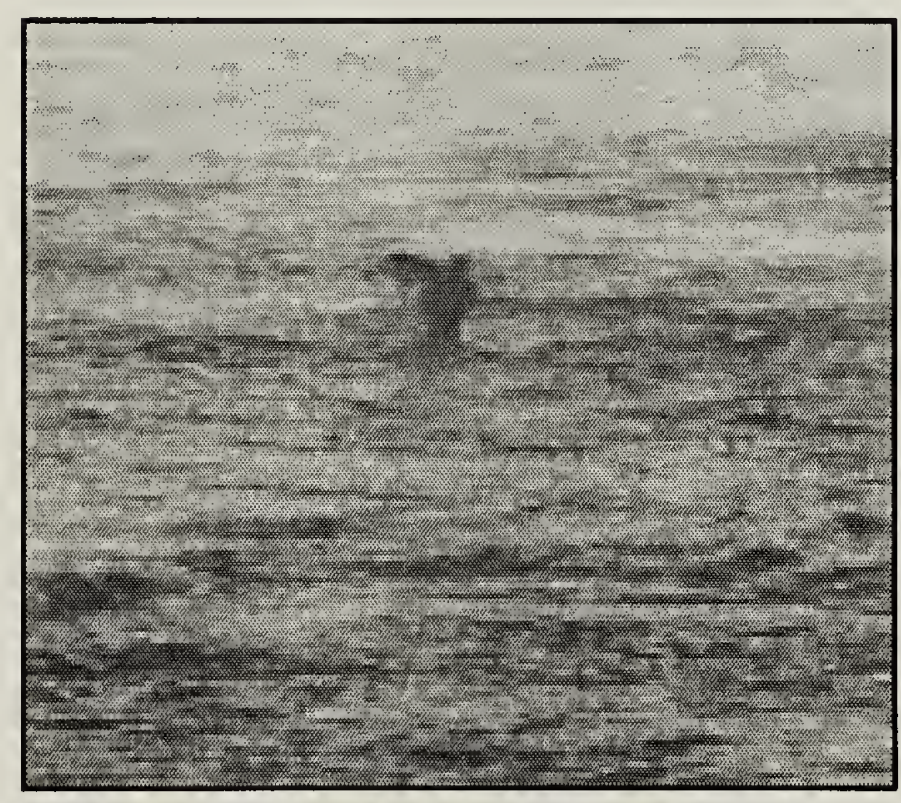

\section{American Dipper photographed on the Eastend Count Robert Gebhardt}

\section{Other rarities}

For the second year in a row the Sandhill Crane made an appearance on the count. Two birds were seen during the count period in the Cabri area. Eurasian Collared-Doves continue to increase with 18 at Moose Jaw. Ten McCown's Longspurs on the Coronach count were only the second winter sighting for this species.

[Editors' note: The increasing number of counts each year reflects the dedication of many committed people and this is very gratifying. It also means that tabulating the count results takes an increasing amount of time. Compilers can help by getting count results to $\mathrm{Al}$ Smith as early as possible. Count results that arrive as late as February cause serious delays to preparation of the count results for publication. To facilitate getting the March issue of Blue Jay out on time, January 31 has been established as the cut off date; counts that arrive on Al Smith's desk after this date will not be included in the March issue tabulations. It is also a great help to the tabulators when compilers submit results on the form they provide. Many hours of work go into creating the tables of count results and your help is these matters is greatly appreciated by the editors, tabulators and Blue Jay readers.]

\section{Count areas and participants}

(Names of compilers are in italics).

1. ARCHERWILL. Bill Carlson, Sharren Carlson, Joe Cooper, Joyce Cooper, Doris Dewhurst, Joanne Folstad, Ken Folstad, Gerald Hiron, Susan Hiron, Pauline Hnetka, Elaine Hughes, Dorothy Klettberg, Willie Klettberg, Judy Revoy, Stan Revoy.

2. ARMIT. Val Harris, Sheila Lamont, Joyce Zak.

3. BANGOR. Allan Bolton, Lynn Bolton, John Maddaford, Leslie Maddaford.

\section{BETHUNE-DILKE. Dong Laing, Vera Laing.}

5. BIGGAR. Dale Booth, Mark Pickett, Linda Schnedar, Lorne Sutherland, Brandon Wapple, Guy Wapple, Marguerite Wapple, Rob Wapple, Sandra Wapple, Ann Weekes.

6. BIRCH HILLS. Carman Dodge, Margaret Mareschal, Moe Mareschal, Bob Mascho, Gayle Mascho, Don Weidl.

7. BRIGHTWATER RESERVOIR. Blanca Xiomara Mora Alvarez, Keith Hobson, Doug Peters, Alan Smith.

8. BROADVIEW. David Chaskavich, Barbara Weidl, Don Weidl, Lorraine Weidl, Tony Weidl.

9. BROMHEAD. Martin Bailey, Carol Bjorklund, John Whitell.

10. CABRI. Don Weidl, Carman Dodge (nonparticipating compiler).

11. CANDLE LAKE. Carman Dodge, Moe Mareschal, Don Weidl (non-participating compiler). 
12. CHERRY LAKE. Karen Herriot, Trevor Herriot, Lorran Wild, Mike Wild.

13. CHURCHBRIDGE. Elin Johnson, Ron Johnson, Wally Karan, Elain Pollock, John Pollock, Ed Wirth, Gloria Wirth.

14. CLARK'S CROSSING. Carol Blenkin, Arelene Block, Peter Block, Janny Bos, Lucille Bradatsch, Dave Cook, Louise Cook, Yvonne Cuttle, Lorne Duczek, Debby Farrow, Marilyn Haskins, Nola Kornder, Gordon Korshinsky, Margaret Korshinsky, Kay Krueger, Gerard Lahey, Carol Maccan, Audrey MacKenzxie, Bill MacKenzie, Kylie McRae, Janet McVittie, Daniel Mosterd, Hilda Noton, Linda Schnedar, Jan Shadick, Stan Shadick, Marten Stoffel, Ruth Welsh, Michael Williams, Kay Willson, Phil Will son, Jim Wood.

15. CODETTE LAKE. Dong Boivin, Wanda Boivin, Barb Weidl.

16. CORONACH. Val Harris, Ron Jensen, Colleen Kessler, Sheila Lamont.

17. CRAVEN. Mary Aird, Betty Binnie, Jared Clarke, Jim Cummings, Lewis Draper, Cliff Elliott, Karen Elliott, Ruth Englund, Bob Ewart, Jon Herriot, Trevor Herriot, Paule Hjertaas, Phil Holloway, Gary Howland, Michele Howland, Lauren Mang, Yvonne McKenzie, Barry Mitschke, Kevin Moore, Ron Myers, Curtis Pollock, Jeanette Taylor.

18. CROOKED LAKE. Boyd Metzler, John Pollock.

19. CROOKED RIVER. Catherine Mehler, Margaret Melller, Morley Mehler, Irene Unruh.

20. CYPRESS HILLS PROVINCIAL PARK (Centre Block). John Douglas, Ryan Dudragne, Joan Hodgins, Jeff Jensen, Ron Jensen, Amy Krause, Rick Krause, David Larson, Margaret Larson, Sue McAdam, Melody Nagel-Hisey, Alma Smith.

21. DUCK LAKE. Keith Hobson, Alan Smith, Phil Taylor.

22. DUNDURN. Alan Smith, Don Weidl.

23. EASTEND. Robert Gebhardt, June Higgins, Joan Hodgins, Harvey Johnson, Keith Schwett, Heidi Scott, Mary Thompson.

24. EMMA LAKE. Bob Hutchison, Gwen Hutchison, Deanna Krng, Norman Krug.

\section{ENDEA VOUR. Norman Harris.}

26. ESTERHAZY-HAZELCLIFFE. Barb Lee, Les Lee, Wayne Provick, John Simpson.

27. ESTEVAN. Val Harris, Sheila Lamont, Craig Palmer, Rose Walton, Guy Wapple.
28. ESTUARY NORTH. Cathy Cocks, Dean Francis, Frances Hartsook, Walter Steinley.

29. FENTON. Carman Dodge, Moe Mareschal, Don Weidl.

30. FORT QU'APPELLE. Elizabeth Aitken, James Armstrong, Phyllis Bordass, Doreen Harman, Barbara Hooper, Ronald Hooper, Alice Isfan, Lois Lamontagne, Vic Lamontagne, Jack Lowe, Jean McKenna, Allan Mlazgar, Florence Pearpoint, Ted Snedker, Fred Warren, Elaine Willox.

31. FORT WALSH. Corlaine Gardner, Rob Gardner, Val Harris, Ron Jensen, Susan McAdam, Wilkes Parsonage, Guy Wapple.

32. GARDINER DAM. Greg Fenty, Val Harris, Ron Jensen, Sig Jordheim, Sheila Lamont, Susan McAdam, Marten Stoffel, Guv Wapple, Dan Zazelenchuk.

33. GOOD SPIRIT LAKE. Bill Anaka, Joyce Anaka, Virginia Baron, Willie Baron, Val Edwards, Phyllis McFaddin, Oney Pollock, Ray Riesz, Lloyd Wilson, Marj Wilson, Julia Wiwchar.

34. GOVENLOCK. Val Harris, Ron Jensen, Rick Jones, Sheila Lamont, Susan McAdam, Joe Saville, Guy Wapple.

35. GRASSLANDS NATIONAL PARK. Richard Cherepek, Medea Curteanu, Pat Fargey, Shelley Larson, Brent McDougall, Colin Schmidt, Robert Sissons, Jucmma Thompson.

36. GRAYSON. Carina Helm, Charles Helm, Daniel Helm, John Hickie, Karl Zimmer.

\section{GRENFELL. Jim Elliott.}

38. HARRIS. Betty Selsey, Fred Selsey, Brandon Wapple, Guy Wapple, Rob Wapple.

39. HEPBURN. Phyllis Siemens.

40. HUDSON BAY. Bill Adam, Monique Cadrain, Jolm Daisley, Doreen Dobrinski, Terry Hansen, Adas Hawrish, Les Hayes, Agnes Lewellin, Brett Lewis, Scott Mackie, Amy Nagus, Laurie Nielsen, Ryley Serhan, Terry Serhan, Ron Shepard, Lee Smith, Wayne Thacker, Ernest Umpherville, John Zolkavich.

41. INDIAN HEAD. Gerry Beattie, Jan Beattie, Melamie Beattie, Amanda Beaulieu, Daniel Beaulieu, Denise Beaulieu, Graeme Beaulieu, Heather Beaulieu, Victor Beaulieu, Chris Blair, Bruce Bottomley, Mel Brayford, Sheila Brayford, Peter Coghlin, Betty Duran, Mike Duran, Eileen Escott, Irv Escott, David Gehl, Laurie Geremia, Roger Geremeia, Lansley Gibbens, Roy Hearn, Sharon Hearn, Gord Howe, Jennifer Jinks, Jim Jinks, Linda Jinks, Joyce Keslering, Richard Keslering, Nick Kucharan, Sheril Kucharan, Dan Loran, Rosalei Namath, Dora Nichols, Pat Nichos, Bob Nolan, Gladys Nolan, Jim Ollett, Marg Ollett, Darlene Redding, Tom 
Redding, Ann Robb, Lorne Scott, Chris Skinner, Fred Skinner, Linda Skinner, Rodney Soron, Donna Thompson, Ronald Thompson, Ellen Varley, Jack Varley, Frank Varesh, Shilrey Walker, Spence Walker, Anne Willerth, Gord Willerth, Elaine Williamson, Jackie Willoughby.

42. KAMSACK. Agnes Beitz, Marlon Brock, Fred Chernoff, Norma Chernoff, Jeff Conley, Nell Conley, Lindee Dewores, Pat Fisher, Bill Fraser, Helen Jellson, Russ Jellson, Carol Koeningbaur, Rudy Koeningbaur, Bernie LaRocque, Laura Leoppkey, Sidney Rielkoff, Isabel Ritchie, Dave Severson, Elsie Severson, Joan Smandych, Joyce Unlow.

43. KELVINGTON (Roscommon School District). Pat Finnie, Diamne Sloan, Marguerite Sloan.

44. KENASTON. Lawrence Beckie.

45. KENOSEE LAKE. Boyd Metzler, Jolın Pollock.

46. KETCHEN NORTH. Dallas Fairburn.

47. KILWINNING. Ed Driver; Margaret Driver.

48. KINDERSLEY NORTH. Jean Harris, Keith Harris.

49. KINISTINO (HORSESHOE BEND). Verma Messer, Dannelle Messer, Doris Verreault, Hubert Verreault.

50. KINLOCH. Elaine Asbjournhus, Wayne Fletcher, Don Forbes, Doreen Forbes, Cliff Logan, Doreen Wickstrom.

51. KUTAWAGAN LAKE. Val Harris, Sheila Lamont.

52. KYLE. David Hamilton, Sig Jordheim, Darryl Jordheim, Jim Lewis, Gordon Nelson, Yvonne Nelson, Glen Pederson, Dan Zazelenchuk.

53. LA RONGE (North). Dianne Allen, Sharon Feschuk, Fred Guerin, Peter Mizanski, Kent Pointon, K. Lynn Riese, Alice Robert, John Schisler, Jan Shewchuk.

54. LAST MOUNTAIN LAKE N.W.A. Val Harris, Kerry Hecker, Ron Kennedy, Sheila Lamont, Lowell Strauss, Lois Vanthuyne.

\section{LEADER NORTH. Daisy Meyers.}

56. LITTLE MANITOU LAKE. John Dunlop, Alan Smith, Rob Wapple.

57. LOVE - TORCH RIVER. Carol Blenkin, Bert Dalziel, Duke Dalziel, Joan Dalziel, Kari Dalziel, Sara Dalziel, Scott Edwards, Rosanne Kirkpatrick, Eileen L'Heureux, Paul L'Heureux, Jesse LcCuyer, Kyla LcCuyer, Bill Matthews, Lynn Matthews, Lillian Nesset, David Pratt.
58. LUSELAND. Joe Brice, Estelle Finley, Graeme Finley, Kim Finley, Liam Finley, Robert Finley, Valerie Finley, Bill Frey, Brent Honeker, Joan Onerheim.

59. MACDOWALL. Hazel Barton, Myron Barton, Carman Dodge.

60. MACNUTT. John Skinner, Tim Wendell.

\section{MATADOR. Cindy Romo, Jim Romo.}

\section{MAYVIEW. Judith Graham, Lisa Rolston.}

63. MEADOW LAKE. Bill Caldwell, Don McGinnis, Linda McGinnis, Joe Twidale, Lorraine Twidale, $B o b$ Wilson, Ian Wilson.

64. MOOSE JAW. Jack Adkins, Elsie Adkins, Edith Bell, Domna Cork, Ron Cork, Fern Dowse, Al Gurnsey, Karry Hanley, Eve King, Leith Knight, Allan Lemieux, Shirley Lemieux, Dolly Macklin, Roger Macklin, Bill McDonald, Lyn McDonald, Len Mowchenko, Jean Ann Mowchenko, Fred Nash, Peter Norys, Helen Norys, Don Parr, Diane Richardson, Ken Richardson, Gus Sagal, Elizabeth Travis, Matt Travis.

65. MOOSE MOUNTAIN. Greg Bobbitt, Bob Cameron.

66. MORSE. Ann Francis, Mike Francis, Randy McCulloch, Joel Priebe, Ken Priebe, Myrna Priebe, Lori Wilson.

67. NIPAWIN. Carol Blenkin, Vi Budd, Harry Budd, Joyce Christiansen, Bert Dalzeil, Sara Dalzeil, Betty Dolman, Shirley Harstad, Wally Harstad, Jennette LeCuyer, Donna Mollberg, Wally Mollberg, Doug Phillips, Peter Phillips, Shane Phillips, Shirley Phillips.

68. NISBET FOREST, NORTHWEST. Ralph Aubrey, Sandra Jewell.

69. NISBET FOREST, WEST. Kim Clark, Kiri Clark, Shamara Clark, Suzanne Clark, Guy Levesque, Patty Levesque, Evelyn Marshall, A1 Scarrow.

70. PIKE LAKE. Tony Allen, Lawrence Beckie, Carol Blenkin, John Bond, Megan Brown, David Cook, Louise Cook, Jill Cornford, Peter Cornford, Yvonne Cuttle, Lorne Duczek, Betty Ann Dunlap, Peter Flood, Bob Girvan, Mike Gollop, Gerard Lahey, Carol Maccan, Margaret Massey, Ted Melville, Daniel Neves, Hilda Noton, Keith Pahl, Frank Roy, Janet Sedgewick, Jaswant Singh, Angela Stern, Marten Stoffel, Mercedes Wayman, Colette Wheler, Michael Williams.

71. PREECEVILLE. Florence Reber, Muriel Mitchell, Mae Tunbridge.

72. PRINCE ALBERT. Jim Bahr, John Burt, Pamela Burt, Kim Clark, Kiri Clark, Shamara Clark, Carman Dodge, Keith Dodge, Millie Fillmore, Ruby Harrington, Doug Hodgins, Sandra Jewell, Rae Ann 
Krug, Murray Little, Michael Newman, Sheryl Newman, Christine Rye, John Rye, Don Weidl, Shyla Wilkinson, Travis Wilkinson.

73. PRINCE ALBERT NATIONAL PARK. Doreen Collingwood, Lorrie Collingwood, Dan Frandsen, Judy Frandsen, Doug Gullickson, Fiona Moreland, Eva Paul, Norm Stolle.

74. QU'APPELLE VALLEY DAM. Carol Blenkin, Carol Maccan, Frank Roy, Jan Shadick, Stan Shadick, Marten Stoffel, Michael Williams, Jim Wood.

\section{RAYMORE. Val Harris, Sheila Lamont.}

76. REGINA. Dan Beveridge Jr, Dan Beveridge Sr, Lionel Bonneville, Stephane Bonneville, Jared Clarke, Bob Ewart, Sandra Ewart, Lawrence Fern, Shirley Friel, Shirley Gerlach, Susan Graham, Jon Herriot, Karen Herriot, Kate Herriot, Trevor Herriot, Dale Hjertaas, Paule Hjertaas, Elaine Hopfner, Phyllis Ilsley, Rhéal Laroche, Bob Luterbach, Lauren Mang, Glen McMaster, Catherine Parkinson, Wayne Pepper, Brian Rainey, Ed Rogers, Margaret Skeel, Frank Switzer, Steven Weir.

77. ROULEAU. Karen Aulie, Vivian Fortman, Marla Gellvear, Kathy Rasmussen, Jeb Sawatsky, Maureen Sawatsky, Patty Spalding, Pat Sterzuk, Linda Thome, Sidni Thome.

78. ROUND LAKE (Qu'Appelle Valley). Dong Francis, Laura Grimeau, Boyd Metzler, John Pollock.

79. SALTCOATS. Bill Anaka, Joyce Anaka, Irene Bobyk, Arden Bradford, Joan Farquharson, Walter Farquharson, Jim Jowsey, Shirley Jowsey, George Maben, Rob Wilson.

80. SASKATCHEWAN LANDING PROVINCIAL PARK. Val Harris, Ron Jensen, Sig Jordheim, Sheila Lamont, Jim Lewis, Sne McAdam, Dan Zazelenchuk.

81. SASKATCHEWAN RIVER FORKS. Carman Dodge, Keith Dodge, Don Weidl (non-participating compiler).

82. SASKATOON. Bruce Acton, Jennifer Acton, Joan Acton, Don Adams, Darlene Aikman, Ray Aikman, Bill Albritton, Elizabeth Albritton, Erin Albritton, Nancy Allan, Tony Allen, Juhachi Asai, Kengo Asai, Sumiko Asai, Neva Bayliss, Judith Benson, Jim Beverage, Mark Bidwell, Janny Bos, Eveline Boudreau, Annemarie Buchmann, Attila Chanady, Mike Chorney, Dave Cook, Paul Coutu, Vi Coutu, Ewen Coxworth, Paul Craig, Yvonne Cuttle, Eileen Daglish, Lorne Duczek, Ann Dzus, Glen Edison, Denis Edmonds, Randi Edmonds, Melanie Elliott, Helen Fast, Joan Feather, David Forbes, Joe Fry, Cathy Fry, Mary Gilliland, Cedric Gillott, Mike Gollop, Jean Gordon, Bob Green, Anne Hanbidge, Helen Hanbidge, John Hanbidge, Marlene Hanbidge, Colleen Hardie, Peter Hardie, Maxine Harriman, Kaija Harris,
Erling Hertzum-Larsen, Grethe Hertzum-Larsen, Cathy Holtslander, Mary Houston, Stuart Houston, Andrea Hull, Kathryn Hull, Peter Hull, Richard Kerbes, Elaine Konkin, Fred Konkin, Gordon Koshinsky, Margaret Koshinsky, Gerard Lahey, Catherine Laratte, Anna Leighton, Ted Leighton, Irene Logan, Carol Maccan, Audrey MacKenzie, Bill MacKenzie, David Miller, Kathryn Miller, Larry Mitchell, Maxine Morrison, Hilda Noton, Keith Pahl, Alison Philips, Dorothy Reimer, Paul Riemer, Marella Rosta, Mary Jean Roy, Andy Salisbury, Craig Salisbury, Lorreine Salisbury, Robert Salisbury, Ariel Santana, Trish Santo, Marjorie Scharf, Murray Scharf, Doug Schmeiser, Laurie Anne Slinger, Alan Smith, Janice Solem, Marten Stoffel, Katie Thorpe, Mary Toews, Hilda Voth, Heather Wagg, Shirley Wedgwood, Ruth Welsh, George West, Shirley West, Joyce Wilkinson, Michael Willicmls, Jim Wood, Stan Woynarski, David Wright, Lynette Wright, Norman Zlotkin.

83. SHAMROCK. Donna Heinrichs, Gladys Heinrichs, Jill Heinrichs, Hngl Hemy.

84. SKULL CREEK. Alyson Bennetto, Erin Bennetto, Ray Bennetto, Sharon Bennetto,

85. SNOWDEN. Eleanor Bodnaruk, Ann Boehler, Ella Butterfield, Lorne Buttterfield, Dale Debler, Sylvia Debler, Diane Friesen, Don Friesen, Irene Hagel, Ernie Hall, Audrey Holliday, Bob Olsen, Jeanette Olsen, Jack Pickett, Karen Priestley, Vera Schemenauer, Orville Schwetz, Vicky Schwetz, Bev Smears, Walter Stevenson, Irene White, Les White, Marleen White.

86. SOMME. Edwin Billeter, Deon Black, Wesley Black, Edwin Bremer, Irene Bremer, Florence Chase, Mike Grywacheski, Barbara Hooper, Margaret Hooper, Ronald Hooper, Gordon Johnson, Elsie Kerr, Jim Martin.

\section{SPINNEY HILL. Ed Ditiver; Margaret Driver.}

88. SPRUCE HOME, SW. Sandra Jewell, Beth Neal, Dale Neal.

89. SQUAW RAPIDS. Val Harris, Sheila Lamont, Marten Stoffel, Guy Wapple.

\section{STENEN. Frances Bury, Peter Buryk.}

91. SWIFT CURRENT. Evelyn Anderson, Trish Ayrey, James Beatie, Jackie Bolton, Laurent Dudragne, Mary Ann Dudragne, Ryan Dudragne, Kathie Hagman, Leonard Head, Hugh Henry, Jeff Jensen, Julie Jensen, Ron Jensen, Vera Lynn Knipfel, Dorine Kreuger, Walter Kreuger, Kim Lamb, Ken Lieir, Darleen Nast, Joe Nast, Ed Nein, Leona Nein, Chet Neufeld, Beth Parchman, Jaynet Payne, Ted Philipchuk, Matthew Smith, Myra Smith, Jared Stephenson, John Stephenson, Irene Stinson, Dan Sawatsky, Dillion Sawatsky, Aaron Thninglestad, Joe Unger, Dave Zacharias, Verdella Zacharias. 
92. TOGO. Donna Dewores, Barb Elsasser, Doug Elsasser, Edith Mann, Helen Tomochko.

93. TORCH RIVER VALLEY. Ken Blaine, Lorna Blaine, Sylvia Fall, Donalda Kreig, Ron Kreig, George Lidster, Jean Lidster, Dale Nelson, Jeannette Nelson, Beatrice Runn, Dave Smith, Karen Smith, Holly Toews, Ruben Toews.

\section{TURTLE LAKE. Merle Robinson.}

95. TURTLEFORD. Brent Keen, Marg Uhlig, Hannah Warrington, David Woof.

96. WEYBURN. Martin Bailey, Leo Belanger, Audrey Bennett, Fred Bennett, Carol Bjorklund, Greg Bobbitt, Bob Cameron, Glen Fleming, Millie Fleming, Fred Garner, K. Kendrick, Doyle Thomas, Val Thomas, Dorothy Whitell, John Whitell.
97. WHITE BEAR. Darryl Jordheim, Floyd Jordheim, Sig Jordleim.

98. WHITEWOOD. Butch Ashfield, Cindy Ashfield, Joe Ashfield, Paul Ashfield, Tom Ede, Wilfred Jordens, Bernice Juzyniec, Felix Juzyniec, Mavis Kay, Ron Kittler, Joyce Kydd, Florence Luhtala, Doreen McPhail, Jean Meadows, Ila Meszaros, Boyd Metzler, Donna Mohr, Brenda Pollock, John Pollock, Florence Pritchard, Lenore Santo, Carol Sawatzke, Diane Veresh, Pat Ward, Jean Wilson.

99. YORKTON. Bill Anaka, Joyce Anaka, Cliff Dixon, Mavin Fairclough, Cheryl Fraser, Norm Fraser, Bob Graham, Elinor Hjertaas, Jim Jowsey, Shirley Jowsey, Harley Large, Ruth Large, Bob Lavender, Sheila Lavender, Janet Lee, George Mabin, Regina Melnyk, Joyce Muir, Dorothy Skene, Geoff Smith, Ruth Smith, Harold Wilkinson, Lloyd Wilson, Marj Wilson.

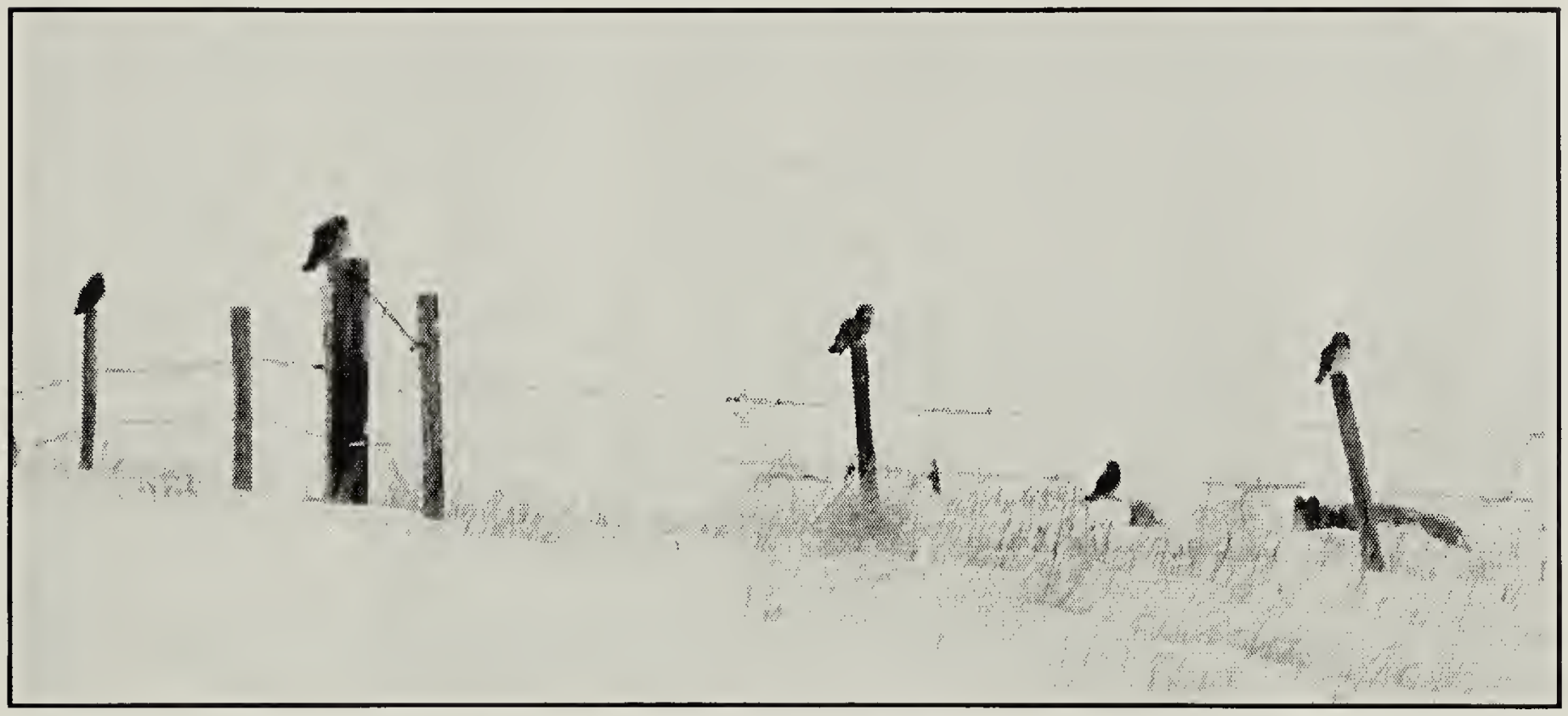

Jamuary 8, 2005, 9 miles north and 4 miles west of Kyle. There are eight Short-eared Owls in the picture and there were seventeen owls at this site that day.

Dan Zazelenchuk

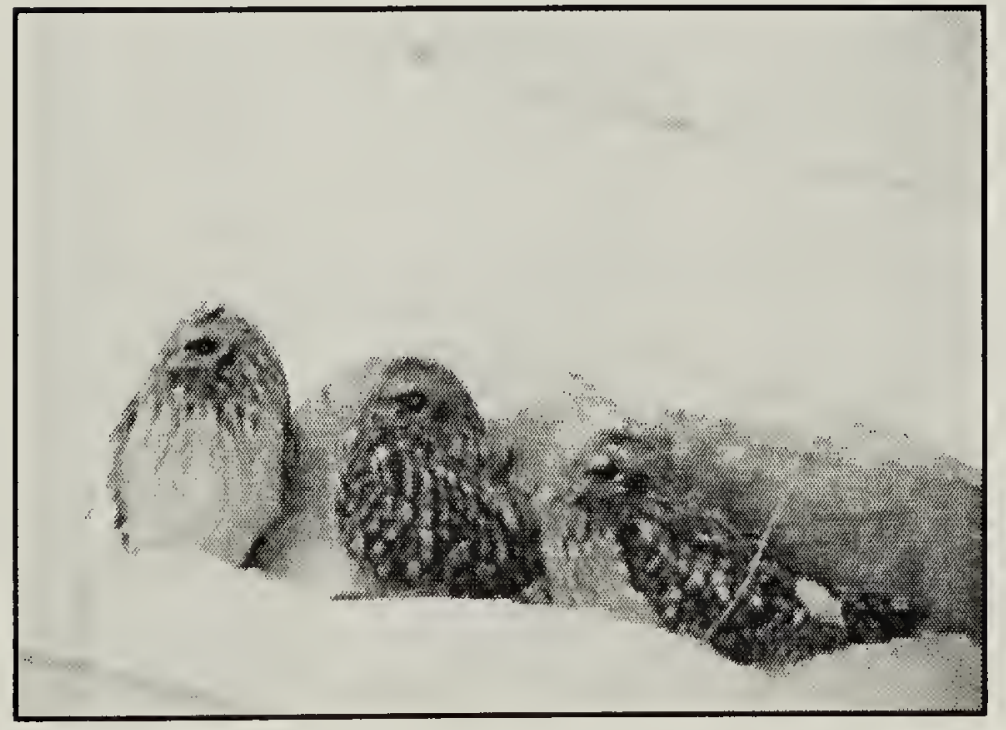

Three Short-eared Owls that are sitting by the rock on the right side of the group picture above.

Dan Zazelenchuk 


\begin{tabular}{|c|c|c|c|c|c|c|c|c|c|}
\hline Locality & Date & 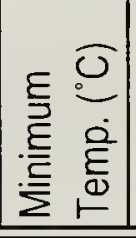 & 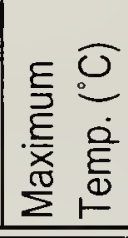 & 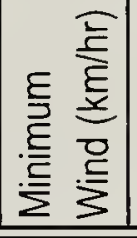 & 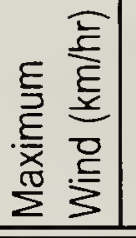 & 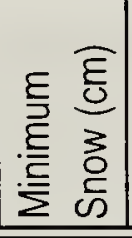 & 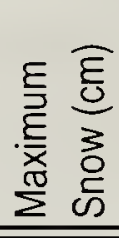 & Sky A.M. & Sky P.M. \\
\hline \multirow{5}{*}{$\begin{array}{l}\text { Archerwill } \\
\text { Armit } \\
\text { Bangor } \\
\text { Bethune-Dilke } \\
\text { Biggar }\end{array}$} & Dec 20 & -25 & -23 & 40 & $40 \mid$ & 5 & 5 & partly cloudy & snowing \\
\hline & $\operatorname{Jan} 4$ & -24 & -21 & 5 & 10 & 15 & 25 & & clear \\
\hline & $\operatorname{Jan} 10$ & -32 & -20 & 8 & 12 & 45 & 50 & partly cloudy & clear \\
\hline & $\operatorname{Jan} 9$ & -20 & -20 & & 8 & & & & clear \\
\hline & $\operatorname{Dec} 27$ & -20 & -15 & 2 & 4 & 5 & 10 & overcast & mostly clear \\
\hline \multirow{5}{*}{$\begin{array}{l}\text { Birch Hills } \\
\text { Brightwater Res. } \\
\text { Broadview } \\
\text { Bromhead } \\
\text { Cabri } \\
\end{array}$} & Dec 18 & -22 & -18 & 1 & 3 & 10 & 15 & overcast & overcast \\
\hline & $\operatorname{Dec} 23$ & -25 & -20 & 0 & 15 & 0 & 10 & clear & partly cloudy \\
\hline & Dec 24 & -15 & -9 & 10 & 10 & 1 & 2 & overcast & overcast \\
\hline & Dec 19 & -10 & 5 & 3 & 6 & 0 & 0 & partly cloudy & cloudy \\
\hline & $\operatorname{Dec} 27$ & -27 & -11 & 0 & 0 & 10 & 10 & clear & clear \\
\hline \multirow{5}{*}{$\begin{array}{l}\text { Candle Lake } \\
\text { Cherry Lake } \\
\text { Churchbridge } \\
\text { Clark's Crossing } \\
\text { Codette Lake }\end{array}$} & $\operatorname{Jan} 2$ & -20 & -19 & 0 & 1 & 15 & 15 & overcast & overcast \\
\hline & $\overline{\operatorname{Jan} 2}$ & -24 & & 22 & 22 & & & & overcast \\
\hline & $\operatorname{Jan} 4$ & -26 & & 2 & 5 & & & & clear \\
\hline & Dec 18 & -17 & -15 & 11 & 18 & 5 & 10 & cloudy & partly cloudy \\
\hline & $\tan 2$ & -24 & -20 & 6 & 11 & 20 & 30 & overcast & overcast \\
\hline \multirow{5}{*}{$\begin{array}{l}\text { Coronach } \\
\text { Craven } \\
\text { Crooked Lake } \\
\text { Crooked River } \\
\text { Cypress Hills PP } \\
\end{array}$} & $\tan 2$ & -26 & -24 & 0 & 5 & 5 & 25 & partly cloudy & mostly clear \\
\hline & Dec 18 & -15 & & 20 & 20 & & & partly cloudy & partly cloudy \\
\hline & $\operatorname{Dec} 17$ & 4 & 7 & 35 & 75 & 0 & 10 & clear & overcast \\
\hline & Dec 29 & -20 & -15 & 0 & 의 & & & clear & clear \\
\hline & Dec 29 & -4 & 2 & 0 & 1 & 5 & 15 & mostly clear & mostly clear \\
\hline \multirow{5}{*}{$\begin{array}{l}\text { Duck Lake } \\
\text { Dundurn } \\
\text { Eastend } \\
\text { Emma Lake } \\
\text { Endeavour } \\
\end{array}$} & Dec 21 & -25 & -15 & 0 & 0 & 10 & 20 & mostly clear & overcast \\
\hline & $\operatorname{Jan} 5$ & -30 & -20 & 8 & 8 & 5 & 10 & clear & clear \\
\hline & $\operatorname{Jan} 3$ & -19 & -11 & 4 & 15 & 10 & 40 & partly cloudy & mostly clear \\
\hline & Dec 17 & 0 & 3 & 30 & 55 & 6 & 10 & partly cloudy & cloudy \\
\hline & Dec 26 & -20 & -15 & 3 & 15 & 18 & 26 & light fog & overcast \\
\hline \multirow{5}{*}{$\begin{array}{l}\text { Esterhazy } \\
\text { Estevan } \\
\text { Estuary North } \\
\text { Fenton } \\
\text { Fort Qu'Appelle } \\
\end{array}$} & $\operatorname{Dec} 18$ & -22 & -13 & 12 & 19 & & & overcast & \\
\hline & $\operatorname{Jan} 1$ & -20 & -18 & 30 & 40 & 25 & 30 & overcast & overcast \\
\hline & \begin{tabular}{|l}
$\operatorname{Jan} 2$ \\
\end{tabular} & -26 & -22 & 2 & 3 & 13 & 18 & clear & clear \\
\hline & Dec 17 & 2 & 6 & 0 & 25 & & 4 & overcast & overcast \\
\hline & $\operatorname{Dec} 18$ & -6 & -4 & 5 & 10 & 2 & 3 & mostly clear & partly cloudy \\
\hline \multirow{5}{*}{$\begin{array}{l}\text { Fort Walsh } \\
\text { Gardiner Dam } \\
\text { Good Spirit Lake } \\
\text { Govenlock } \\
\text { Grasslands NP } \\
\end{array}$} & Dec 18 & -2 & 5 & 0 & 3 & 0 & 15 & partly cloudy & cloudy \\
\hline & Dec 20 & -20 & -12 & 5 & 6 & 0 & 3 & cloudy & overcast \\
\hline & Dec 29 & -20 & -14 & 2 & 5 & 15 & 22 & clear & clear \\
\hline & $\operatorname{Dec} 19$ & -5 & -3 & 70 & 80 & 0 & 2 & mostly clear & mostly clear \\
\hline & $\operatorname{Jan} 4$ & -32 & -25 & 0 & 5 & 10 & 30 & partly cloudy & partly cloudy \\
\hline \multirow{5}{*}{$\begin{array}{l}\text { Grayson } \\
\text { Grenfell } \\
\text { Harris } \\
\text { Hepburn } \\
\text { Hudson Bay West }\end{array}$} & Dec 22 & -28 & -20 & 0 & 20 & 0 & 5 & partly cloudy & partly cloudy \\
\hline & Dec 26 & -16 & -12 & 10 & 10 & 10 & 10 & overcast & overcast \\
\hline & Dec 31 & -20 & -18 & 4 & 6 & 0 & 15 & overcast & overcast \\
\hline & Dec 14 & -10 & -2 & 2 & 5 & 5 & 10 & mostly clear & mostly clear \\
\hline & $\overline{\operatorname{Dec} 30}$ & -23 & -20 & 20 & 25 & 25 & 30 & cloudy & \\
\hline \multirow{5}{*}{$\begin{array}{l}\text { Indian Head } \\
\text { Kamsack } \\
\text { Kelvington } \\
\text { Kenaston } \\
\text { Kenosee Lake } \\
\end{array}$} & & & & & & & & & \\
\hline & & very & cold & very & windy & & 61 & overcast & \\
\hline & Dec 29 & -15 & & & 10 & & 15 & partly cloudy & clear \\
\hline & Dec 18 & -15 & -15 & 17 & 17 & & 2 & partly cloudy & mostly clear \\
\hline & Dec 29 & -10 & -6 & 0 & 1 & 10 & 25 & clear & mostly clear \\
\hline \multirow{5}{*}{$\begin{array}{l}\text { Ketchen North } \\
\text { Kilwinning } \\
\text { Kindersley North } \\
\text { Kinistino } \\
\text { Kinloch } \\
\end{array}$} & Dec 26 & -23 & -16 & 3 & 15 & 20 & 28 & cloudy & light snow \\
\hline & Dec 18 & -21 & -16 & 5 & 15 & 4 & 21 & clear & clear \\
\hline & $\operatorname{Dec} 27$ & -8 & 13 & & 2 & & 5 & clear & clear \\
\hline & $\operatorname{Jan} 2$ & -22 & -22 & 2 & 3 & 12 & 12 & clear & clear \\
\hline & $\operatorname{Jan} 3$ & -24 & -20 & 5 & 10 & 23 & 25 & mostly clear & mostly clear \\
\hline
\end{tabular}


Table 1-2 Weather and Conditions

\begin{tabular}{|c|c|c|c|c|c|c|c|c|c|}
\hline Locality & Date & 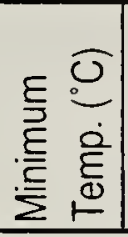 & 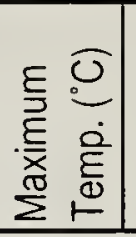 & 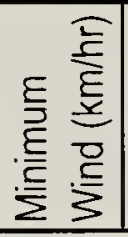 & 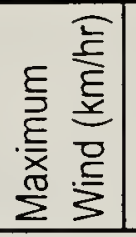 & 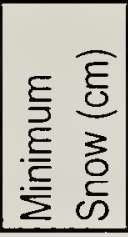 & 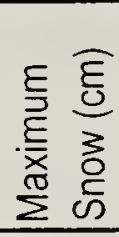 & Sky A.M. & Sky P.M. \\
\hline \multirow{5}{*}{$\begin{array}{l}\text { Kutawagan Lake } \\
\text { Kyle } \\
\text { LaRonge North } \\
\text { Last Mountain Lake } \\
\text { Leader North } \\
\end{array}$} & Dec 25 & -18 & -16 & 10 & 20 & 10 & 20 & overcast & overcast \\
\hline & $\operatorname{Jan} 3$ & -28 & -18 & 0 & 18 & 5 & 15 & overcast & overcast \\
\hline & $\operatorname{Dec} 30$ & -20 & -18 & 10 & 15 & & 60 & overcast & partly cloudy \\
\hline & $\operatorname{Dec} 30$ & -20 & -18 & 20 & 40 & 10 & 25 & overcast & overcast \\
\hline & $\operatorname{Dec} 29$ & -25 & -4 & 0 & 0 & 5 & 10 & clear & clear \\
\hline \multirow{5}{*}{$\begin{array}{l}\text { Little Manitou Lake } \\
\text { Love-Torch River } \\
\text { Luseland } \\
\text { MacDowall } \\
\text { MacNutt }\end{array}$} & Dec 29 & -21 & -13 & 0 & 2 & 5 & 20 & fog & partly cloudy \\
\hline & $\operatorname{Dec} 27$ & -33 & -22 & 0 & 20 & 12 & 26 & overcast & overcast \\
\hline & $\operatorname{Jan} 5$ & -28 & -14 & 5 & 20 & 5 & 25 & mostly clear & mostly clear \\
\hline & $\operatorname{Jan} 1$ & -22 & -18 & & 15 & 10 & 12 & overcast & overcast \\
\hline & $\operatorname{Dec} 20$ & -11 & & & 15 & 15 & 20 & clear & clear \\
\hline \multirow{5}{*}{$\begin{array}{l}\text { Matador } \\
\text { Mayview } \\
\text { Meadow Lake } \\
\text { Moose Jaw } \\
\text { Moose Mountain } \\
\end{array}$} & $\operatorname{Dec} 29$ & -13 & -10 & 0 & 30 & 6 & 12 & clear & mostly clear \\
\hline & Dec 21 & -30 & & 0 & 0 & & 30 & clear & \\
\hline & $\operatorname{Dec} 26$ & -21 & -18 & & 3 & 20 & 35 & mostly clear & mostly clear \\
\hline & $\operatorname{Dec} 27$ & -19 & -13 & & 10 & 2 & 7 & overcast & overcast \\
\hline & $\operatorname{Dec} 22$ & -24 & -19 & 5 & 8 & 0 & 6 & partly cloudy & partly cloudy \\
\hline \multirow{5}{*}{$\begin{array}{l}\text { Morse } \\
\text { Nipawin } \\
\text { Nisbet Forest NW } \\
\text { Nisbet Forest West } \\
\text { Pike Lake }\end{array}$} & $\operatorname{Dec} 23$ & -30 & -5 & 6 & 10 & & 4 & overcast & overcast \\
\hline & Dec 28 & -20 & -18 & 6 & 10 & 10 & 13 & partly cloudy & overcast \\
\hline & $\operatorname{Dec} 31$ & -29 & -15 & & 3 & & 20 & overcast & overcast \\
\hline & $\operatorname{Jan} 1$ & -21 & -19 & 10 & 20 & 10 & 20 & overcast & overcast \\
\hline & $\operatorname{Jan} 2$ & -23 & -18 & 8 & 15 & 12 & 20 & mostly clear & overcast \\
\hline \multirow{5}{*}{$\begin{array}{l}\text { Preeceville } \\
\text { Prince Albert } \\
\text { Prince Albert NP } \\
\text { Qu'Appelle Dam } \\
\text { Raymore }\end{array}$} & $\operatorname{Jan} 1$ & -25 & & & 40 & & 13 & partly cloudy & \\
\hline & Dec 19 & -6 & 0 & 5 & 15 & 3 & 10 & overcast & overcast \\
\hline & Dec 22 & -30 & -28 & 5 & 10 & 30 & 75 & clear & clear \\
\hline & $\operatorname{Dec} 19$ & 2 & 8 & 17 & 40 & 0 & 5 & mostly clear & cloudy \\
\hline & Dec 27 & -20 & -15 & 30 & 40 & 10 & 25 & overcast & overcast \\
\hline \multirow{5}{*}{$\begin{array}{l}\text { Regina } \\
\text { Rouleau } \\
\text { Round Lake } \\
\text { Saltcoats } \\
\text { Sask. Landing PP } \\
\end{array}$} & $\operatorname{Dec} 26$ & -18 & -13 & 13 & 22 & 6 & 15 & cloudy & cloudy \\
\hline & Jan 1 & -25 & -18 & 28 & 35 & 40 & 50 & overcast & overcast \\
\hline & $\operatorname{Dec} 28$ & -14 & -5 & 0 & 5 & & 25 & clear & mostly clear \\
\hline & $\operatorname{Dec} 28$ & -19 & -11 & 1 & 1 & 4 & 10 & mostly clear & mostly clear \\
\hline & Dec 21 & -18 & & & 5 & 0 & 10 & cloudy & cloudy \\
\hline \multirow{5}{*}{$\begin{array}{l}\text { Sask. R. Forks } \\
\text { Saskatoon } \\
\text { Shamrock } \\
\text { Skull Creek } \\
\text { Snowden } \\
\end{array}$} & $\operatorname{Dec} 29$ & -22 & -19 & 0 & 2 & 10 & 10 & clear & clear \\
\hline & Dec 26 & -21 & -19 & 18 & 24 & 10 & 15 & overcast & overcast \\
\hline & $\operatorname{Dec} 18$ & -8 & -6 & 15 & 15 & 0 & 6 & overcast & overcast \\
\hline & Dec 26 & -15 & -10 & 1 & 2 & 5 & 10 & mostly clear & partly cloudy \\
\hline & $\overline{\operatorname{Dec} 31}$ & -24 & -18 & 1 & 1 & 21 & 27 & mostly clear & partly cloudy \\
\hline \multirow{5}{*}{$\begin{array}{l}\text { Somme } \\
\text { Spinney Hill } \\
\text { Spruce Home, SW } \\
\text { Squaw Rapids } \\
\text { Stenen } \\
\end{array}$} & $\operatorname{Dec} 30$ & -20 & -18 & 10 & 14 & 30 & 35 & overcast & overcast \\
\hline & Dec 14 & -3 & 4 & 5 & 15 & 2 & 15 & clear & mostly clear \\
\hline & Dec 19 & -20 & 5 & 0 & 6 & 5 & 15 & & \\
\hline & $\operatorname{Jan} 5$ & -26 & -18 & 30 & 40 & 20 & 25 & mostly clear & mostly clear \\
\hline & Jar 1 & -24 & -22 & 5 & 10 & & 30 & partly cloudy & mostly clear \\
\hline \multirow{5}{*}{$\begin{array}{l}\text { Swift Current } \\
\text { Togo } \\
\text { Torch River Valley } \\
\text { Turtle Lake } \\
\text { Turtleford } \\
\end{array}$} & $\operatorname{Dec} 27$ & -15 & \begin{tabular}{|c|}
-10 \\
\end{tabular} & 0 & 5 & 5 & 10 & overcast & clear \\
\hline & $\operatorname{Jan} 3$ & -24 & -18 & 0 & 10 & 25 & 30 & mostly clear & mostly clear \\
\hline & $\operatorname{Dec} 29$ & -20 & -15 & 0 & & 7 & 9 & mostly clear & mostly clear \\
\hline & $\operatorname{Dec} 21$ & -30 & -25 & 15 & 50 & & 12 & overcast & overcast \\
\hline & $\operatorname{Dec} 29$ & -26 & -18 & 0 & 5 & 4 & 15 & overcast & clear \\
\hline \multirow{4}{*}{$\begin{array}{l}\text { Weyburn } \\
\text { White Bear } \\
\text { Whitewood } \\
\text { Yorkton }\end{array}$} & $\operatorname{Dec} 21$ & -17 & -10 & 2 & 5 & 0 & 0 & partly cloudy & partly cloudy \\
\hline & $\operatorname{Jan} 4$ & -25 & -20 & 0 & 0 & 15 & 20 & clear & clear \\
\hline & $\operatorname{Jan} 2$ & -31 & -20 & 5 & 20 & 30 & 70 & clear & mostly clear \\
\hline & Dec 18 & -20 & -18 & 8 & 15 & 6 & & clear & clear \\
\hline
\end{tabular}




\begin{tabular}{|c|c|c|c|c|c|c|c|c|c|c|c|c|c|c|c|c|c|c|c|c|c|c|c|}
\hline \multirow[b]{2}{*}{ Locality } & \multicolumn{7}{|c|}{ Effort } & \multicolumn{16}{|c|}{ Habitat } \\
\hline & 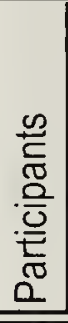 & \begin{tabular}{|c}
$\overline{8}$ \\
L \\
$\overline{0}$ \\
है \\
\end{tabular} & 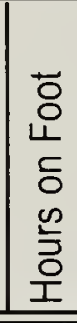 & 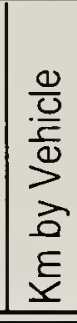 & 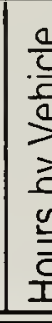 & 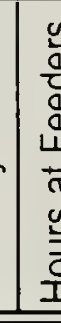 & 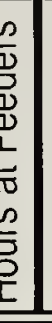 & 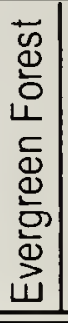 & 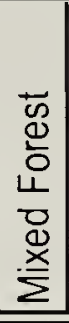 & 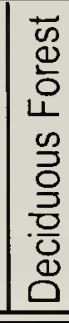 & 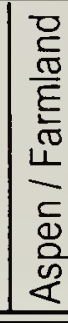 & $\mid \begin{array}{l}0 \\
0 \\
0 \\
0 \\
0 \\
0\end{array}$ & 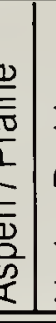 & 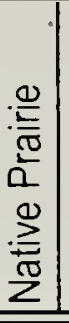 & 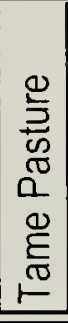 & 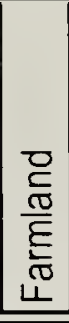 & 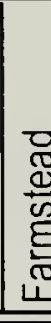 & & $\begin{array}{l}\frac{c}{\pi} \\
\frac{0}{2} \\
\end{array}$ & $\begin{array}{l}\frac{\bar{d}}{\bar{n}} \\
3 \\
\overline{\mathrm{d}} \\
\overline{0}\end{array}$ & 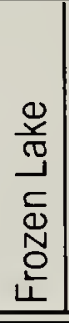 & 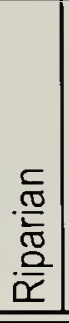 & 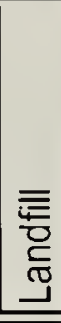 \\
\hline Archerwill & 14 & 3 & 2 & & & & & & 40 & & & & & & & & $\overline{6}$ & $\underline{0}$ & & & & & \\
\hline Armit & 3 & 1 & 1 & 112 & & & 0 & 20 & 30 & 20 & 20 & & & & & & & & 10 & & & & \\
\hline Bangor & 4 & & & 30 & & 5 & & & & & 15 & & 5 & & 10 & & 6 & 0 & & & & & \\
\hline Bethune-Dilke & 2 & & & 40 & & 4 & & & & & & & 1 & 18 & & 80 & & & 1 & & & & \\
\hline Biggar & 10 & 10 & 8 & 478 & 1 & & 7 & & & & 10 & & & & & 57 & & & 33 & & & & \\
\hline Birch Hills & 6 & 8 & 2 & 222 & 1 & & 3 & & & & 30 & & & & 10 & 40 & $\overline{1}$ & 0 & 10 & & & & \\
\hline Brightwater Res. & 4 & 7 & $\overline{3}$ & 160 & & 5 & & & & 15 & & & 10 & 5 & 5 & 30 & 3 & 5 & & 5 & & & \\
\hline Broadview & 5 & 2 & 2 & 142 & & 5 & 3 & & & 20 & 30 & & 10 & 5 & 10 & 10 & 1 & 0 & 5 & & & & \\
\hline Bromhead & 3 & & 1 & & & 6 & & & & & & & & 5 & & 90 & 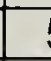 & 5 & & & & & \\
\hline Cabri & 1 & 1 & 1 & 104 & & 7 & & & & & & & & 10 & & 75 & 1 & 0 & 5 & & & & \\
\hline Candle Lake & 2 & & & 134 & 4. & & & 30 & 30 & 30 & & & & & & & & & 10 & & & & \\
\hline Cherry Lake & 4 & 8.0 & 4.0 & 15 & 1. & & & & & & 10 & & 30 & & & & $\overline{1}$ & 0 & & & & & \\
\hline Churchbridge & 6 & & & & & 9 & .0 & & & & & & & & & & 10 & 0 & & & & & \\
\hline Clark's Crossing & 32 & 29 & 19 & 623 & 2 & $8 \longdiv { 6 }$ & .0 & 1 & & 1 & 25 & & 2 & 2 & 1 & 18 & $\overline{3}$ & 0 & 13 & 7 & & & \\
\hline Codette Lake & 3 & 2.0 & 1.0 & 92 & 4. & & & & 5 & & 25 & & & & & 50 & 2 & 0 & & & & & \\
\hline Coronach & 4 & 6.0 & 3.0 & 162 & 8. & & .5 & & & & 5 & & & & & 30 & 1 & 5 & 10 & 40 & & & \\
\hline Craven & 22 & 13 & 4.0 & 345 & 2 & & & & & & 20 & & 20 & & & 30 & 1 & 0 & 20 & & & & \\
\hline Crooked Lake & 2 & 0.5 & 1.0 & 36 & 5. & & & & & 40 & 30 & & & & & 15 & & & & 15 & & & \\
\hline Crooked River & 4 & & & 25 & 2. & & & & 35 & & & & & & & 40 & 2 & 5 & & & & & \\
\hline Cypress Hills PP & 12 & 15 & 8.8 & 50 & 2. & 24 & .0 & 60 & 20 & & & & 10 & 10 & & & & & & & & & \\
\hline Duck Lake & 3 & 10 & 3.0 & 86 & 5. & & & 5 & 35 & 10 & 20 & & & & 5 & 10 & & & 10 & 5 & & & \\
\hline Dundurn & 2 & 1.0 & 0.8 & 110 & 7. & & & & & 10 & 10 & & 10 & 10 & & 40 & & & 20 & & & & \\
\hline Eastend & 7 & 10 & 3.5 & 84 & 4. & & & & 15 & & 60 & & 5 & & & & & 5 & 10 & 5 & & & \\
\hline Emma Lake & 4 & 2.0 & 1.5 & 52 & 5. & \begin{tabular}{l|l}
57 \\
\end{tabular} & .0 & & 95 & & & & & & & & & 5 & & & & & \\
\hline Endeavour & 1 & 5.0 & 5.0 & 26 & 1. & $0 \sqrt{3}$ & .0 & & & & 40 & & & & & & 4 & 0 & 20 & & & & \\
\hline Esterhazy & 4 & & & 12 & 3 & & & & & & 30 & & & & & 70 & & & & & & & \\
\hline Estevan & 5 & 3.0 & 2.5 & 266 & 1 & & & & & $\overline{15}$ & & & & & & 20 & 1 & 5 & 30 & 20 & & & \\
\hline Estuary North & 4 & 5.0 & 3.0 & 75 & 5 & & 0 & & & 70 & & & & 10 & & 15 & 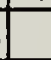 & 5 & & & & & \\
\hline Fenton & 3 & 2.0 & 0.5 & 148 & 5 & & .5 & & & 10 & 50 & & & & 5 & 15 & 1 & 5 & 5 & 1 & & & \\
\hline Fort Qu'Appelle & 17 & 2.0 & 0.5 & 220 & 6. & & 12 & & & 10 & 30 & & & & & 30 & 1 & $\underline{0}$ & 10 & 10 & & & \\
\hline Fort Walsh & 8 & 20 & 16 & 370 & 1 & & & & 61 & & & & & 5 & & 10 & & & & & & 24 & \\
\hline Gardiner Dam & $\overline{9}$ & 10 & 6.3 & 581 & 2 & 5 & & & & & 1 & if & & & & 77 & & & & \begin{tabular}{|l|}
17 \\
\end{tabular} & & 5 & \\
\hline Good Spirit Lake & 11 & 1.0 & 0.5 & 103 & 6 & $5 \sqrt{5}$ & .5 & 5 & & 10 & 10 & & 5 & 5 & 5 & 50 & & 5 & 5 & & & & \\
\hline Govenlock & 7 & 7.5 & 4.3 & 351 & 1 & 4 & & & & & 5 & 5 & & 60 & & 10 & 2 & 5 & & & & & \\
\hline Grasslands NP & 8 & 9.0 & 4.5 & 131 & 6 & & & & & & & & & 65 & 10 & 15 & & & 10 & & & & \\
\hline Grayson & 5 & 4.0 & 1.0 & 60 & 4 & $\begin{array}{ll}0 & 1 \\
\end{array}$ & .0 & & & 5 & 40 & & 10 & & & 20 & 1 & 0 & 5 & 10 & & & \\
\hline Grenfell & & 14 & 3.5 & & & & & & & & & & & & & 5 & & & 95 & & & & \\
\hline Harris & 5 & 4.0 & 2.5 & 267 & 6 & 82 & 0 & & & & 26 & & & & & 57 & & & 19 & & & & \\
\hline Hepburn & 1 & & & 64 & 1 & & .0 & & & & 80 & & & & & & & & 20 & & & & \\
\hline Hudson Bay West & 19 & & & 32 & 1 & 0 & 12 & & 20 & & & & & & & 25 & 2 & 5 & 30 & & & & \\
\hline Indian Head & 58 & & & & & & & & & & & & & & & & & & & & & & \\
\hline Kamsack & 22 & & & & & & & 20 & 5 & & 10 & & & 15 & 10 & & 4 & 0 & & & & & \\
\hline Kelvington & 3 & & & 70 & 3 & & .0 & & & & $\overline{30}$ & & & & & 60 & 1 & 0 & & & & & \\
\hline Kenaston & 1 & & & 110 & 4 & & 0 & & & & & & 13 & & & 80 & & 5 & 2 & & & & \\
\hline Kenosee Lake & 2 & 0.5 & 1.5 & 114 & 3 & $0] 0$ & .5 & & $\underline{5}$ & 40 & 10 & & 5 & & & & & & 40 & & & & \\
\hline Ketchen North & 1 & 0.5 & 2.0 & 50 & 2 & $0]$ & 0 & & & & 30 & & & & 20 & 20 & 2 & 0 & 10 & & & & \\
\hline Kilwinning & 2 & 1.0 & 0.5 & 170 & 6 & 0 & & 10 & 5 & 30 & 60 & & & & 5 & & & & & & & & \\
\hline Kindersley North & 2 & 1.0 & 0.8 & 90 & 4 & $\underline{0}$ & & & & & & & & & & 8 & 3 & 30 & 60 & 2 & & & \\
\hline Kinistino & 4 & 2.0 & 2.0 & & & & .0 & & 30 & & & & & & 40 & & 3 & 30 & & & & & \\
\hline Kinloch & 6 & 9.0 & 6.0 & 90 & 2 & 0.08 & 3 & 20 & 40 & & & & & & & & 4 & 0 & & & & & \\
\hline
\end{tabular}


Table 2-2 Coverage

\begin{tabular}{|c|c|c|c|c|c|c|c|c|c|c|c|c|c|c|c|c|c|c|c|c|c|}
\hline \multirow[b]{2}{*}{ ocality } & \multicolumn{6}{|c|}{ Effort } & \multicolumn{15}{|c|}{ Habitat } \\
\hline & 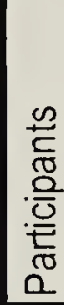 & 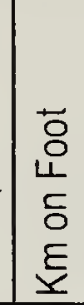 & 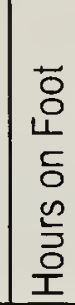 & 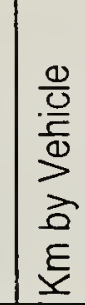 & 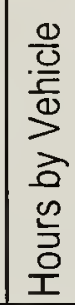 & 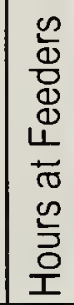 & 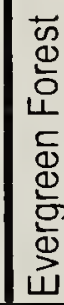 & 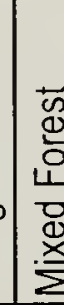 & 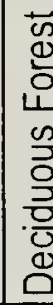 & 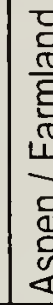 & & 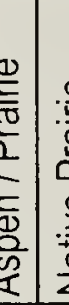 & & 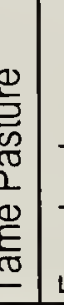 & & & 몬 & 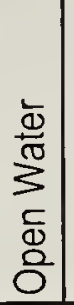 & 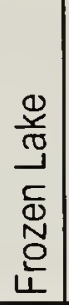 & & \\
\hline$\overline{\text { Kutawagan Lake }}$ & & 2.0 & & 200 & 8.0 & & & & & $\overline{2}$ & & & 10 & & & & 10 & & & & \\
\hline & & 322 & & 501 & 21 & 1.0 & & & & & & 2 & & 5 & 63 & 1 & 1 & & & & \\
\hline LaRonge North & & 8.0 & & & 1.3 & 8.2 & 70 & $\underline{s}$ & 1 & & & & & & & & $c$ & & & & \\
\hline Last Mountain Lake & & 1.0 & & 215 & 8.5 & 0.5 & & & & 1 & & & 30 & & 40 & 10 & 10 & & & & \\
\hline Leader North & & 5.0 & 4.0 & 25 & 2.0 & & & & & & & & $\underline{0}$ & & 40 & 10 & & & & & \\
\hline Little Manitou Lake & & & & 135 & 5.5 & & & & & 2 & & 10 & & & 30 & & 35 & & & & \\
\hline Love-Torch River & 16 & 2.0 & 1.0 & 230 & 12 & 14 & 10 & 11 & & 6 & & & & & & 10 & 10 & & & & \\
\hline Luseland & & 9.0 & & 224 & 7.0 & 4.0 & & & & & 1 & 10 & 10 & 10 & 20 & 10 & 10 & & & & \\
\hline MacDowall & 3 & & & 65 & 3.0 & 3.0 & & 11 & & 8 & & & & & & 10 & & & & & \\
\hline MacNutt & & 10 & 1.0 & 50 & 3.0 & & & & & & & & & & & & 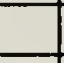 & & & & \\
\hline Matador & & 3.0 & 1.5 & 120 & 4.0 & & & & & & & 24 & 7 & 5 & 1 & & & & & & \\
\hline Mayview & 2 & & & & 0.5 & 2.0 & & 9 & & 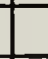 & & & & & 10| & & & & & & \\
\hline Meadow Lake & & 8.0 & 3.5 & \begin{tabular}{|l|l|}
55 \\
\end{tabular} & 3.5 & 5.0 & & 4 & & & & & & & 30 & & 3 & & & & \\
\hline Moose Jaw & 27 & 26 & 18 & 3242 & 21 & 30 & 4 & 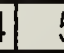 & & & & & $\underline{0}$ & & & 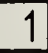 & 80 & & & & \\
\hline Moose Mountain & & 2.0 & 0.6 & 6123 & 4.0 & 0.4 & & & 6 & 2 & & & & & 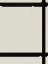 & & 15 & & & & \\
\hline Morse & 7 & 0.5 & 0.3 & $3 \longdiv { 1 5 2 }$ & 4.5 & 0.3 & & & & & & & & & 80 & 10 & 10 & & & & \\
\hline Nipawin & 16 & 3.0 & 1.5 & 160 & 7.0 & 39 & & 2 & & & & & & & 20 & & 50 & $\mid 10$ & & & \\
\hline Nisbet Forest NW & 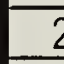 & & & & & 6.8 & & 5 & & & & & & & & 50 & 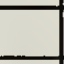 & & & & \\
\hline Nisbet Forest West & 8 & & 0.5 & 5100 & 3.5 & 12 & 10 & & $\underline{3}$ & 1 & & & & & 40 & 10 & & & & & \\
\hline Pike Lake & 30 & 29 & & 632 & 26 & & & & 1 & 1 & & & & 5 & 15 & 15 & 40 & & & & \\
\hline Preeceville & 3 & & & & & 5.0 & & & & & & & & & & 100 & & & & & \\
\hline Prince Albe & 21 & 36 & 15 & 286 & 15 & 1.5 & & & & 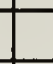 & & 5 & & & & & 85 & 1 & & & \\
\hline Prince Albert NP & 8 & 25 & 7.0 & & & - & 15 & 4 & 2 & & & & & & & & 2 & 15 & & & \\
\hline Qu'Appelle Dam & & 5.0 & & 8400 & 10 & & & & 1 & & & 20 & & 5 & 10 & & 15 & 10 & & & \\
\hline Raymore & & 3.0 & 1.5 & 5188 & 7.5 & 0.5 & & & & 8 & & & & & 10 & & 10 & & & & \\
\hline Regina & 27 & 60 & 23 & $3 \longdiv { 5 3 0 }$ & 22 & 3.0 & & & 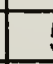 & & & 5 & & 4 & 35 & 12 & 35 & 1 & & & \\
\hline Rouleal & 10 & & & 10 & 2.0 & 6.0 & & & & & & & & & & & 100 & & & & \\
\hline Round Lake & $\underline{4}$ & 2.0 & 1.0 & 221 & 7.5 & 7.0 & & & 4 & 3 & & & & & 20 & & & 10 & & & \\
\hline Saltcoats & 10 & & & 500 & 13 & 3.0 & & & & 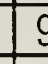 & & & & & & & 5 & & & & \\
\hline Sask. Landing PP & 7 & 11 & 6.5 & $5 \longdiv { 3 9 3 }$ & & & & & & 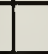 & & 5 & & 15 & 34 & 25 & & 1 & & & \\
\hline Sask. R. Forks & 2 & 23.0 & 2.1 & 80 & 3.2 & & 11 & 1 & 2 & 2 & & 5 & & 5 & 20 & 10 & & & & & \\
\hline Saskatoon & 115 & 102 & 58 & 8948 & 51 & 124 & & & 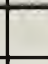 & {$[1$} & & & 1 & & 8 & 15 & 50 & & & & \\
\hline Shamrock & 4 & & & 185 & 10 & & & & & & & & & & 80 & 20 & & & & & \\
\hline Skull Creek & 4 & & & 60 & 4.0 & 8.0 & & & & 1 & & 10 & & $\overline{10}$ & 60 & & & & & & \\
\hline Snowden & 23 & & 1.0 & 91 & 3.0 & 4.0 & & 2 & & & & & & & 20 & 40 & 20 & & & & \\
\hline Somme & 13 & \begin{tabular}{|l|l}
3 & 1.0 \\
\end{tabular} & 1. & 160 & 6.0 & 3.0 & & 3 & 1 & & & & & & 30 & 20 & 10 & & & & \\
\hline Spinney Hill & 2 & 3.0 & 0.8 & 8130 & 5.5 & & & 1 & 4 & & & & 0 & & 20 & & & & & & \\
\hline Spruce Home, SW & 3 & & & & 6.0 & 6.0 & & & & & & & & & 80 & 20 & & & & & \\
\hline Squaw Rapids & $\underline{4}$ & 5.0 & 5.5 & 5328 & 11 & 0.5 & - & 6 & _ & & & & & & 7 & 7 & & 10 & & & \\
\hline Stenen & 2 & & & & & 2.0 & 10 & & 9 & & & & & & & & & & & & \\
\hline Swift Current & 37 & 56 & 2 & 2395 & 26 & 47 & & & & & & & 5 & 5 & 64 & & 10 & & & & \\
\hline Togo & 5 & & & 50 & 2 & & 10 & $\underline{3}$ & 1 & & & & $\overline{0}$ & 10 & 10 & & & & & & \\
\hline Torch River Valley & 12 & 43.0 & 1.0 & \begin{tabular}{c|c}
040 \\
\end{tabular} & & 10 & & 1 & & & & & & & & 90 & & & & & \\
\hline Turtle Lake & 1 & & & 50 & & 9.0 & & 5 & 1 & & & & & & 5 & & 5 & & & & \\
\hline Turtleford & 2 & 431 & 8.1 & 0180 & 6.0 & 4.0 & & & & 7 & & & & & 20 & 10 & & & & & \\
\hline Weyburn & 14 & & & 227 & 11 & 4.5 & & & & & & & 5 & & 82 & & 8 & & & & \\
\hline White Bear & & $3 \longdiv { 4 . 0 }$ & & 114 & & & & & & & & & 20 & & 60 & & & & & & \\
\hline Whitewood & 25 & 53.5 & 3. & 0431 & 12 & 5 & & & & 2 & & & & & & & 75 & & & & \\
\hline Yorkton & 2 & 40.5 & 50. & $5 \mid 323$ & 18 & & & & & & & & & & 40 & & 50 & & & & \\
\hline
\end{tabular}


Figure 1. Location of 2004 counts (numbers correspond to those in text under Count areas and participants).

Natural Vegetation Zones*
A. Subarctic Woodland
B. Northern Boreal Forest
C. Southern Boreal Forest
D. Aspen Parkland
E. Mixed Prairie
F. Dry Mixed Prairie
G. Cypress Hills

*adapted from: Thorpe, J. 1999.

Natural Vegetation. P. 133 in

Atlas of Saskatchewan (K. Fung, ed)

Univ. of Sask., Saskatoon.
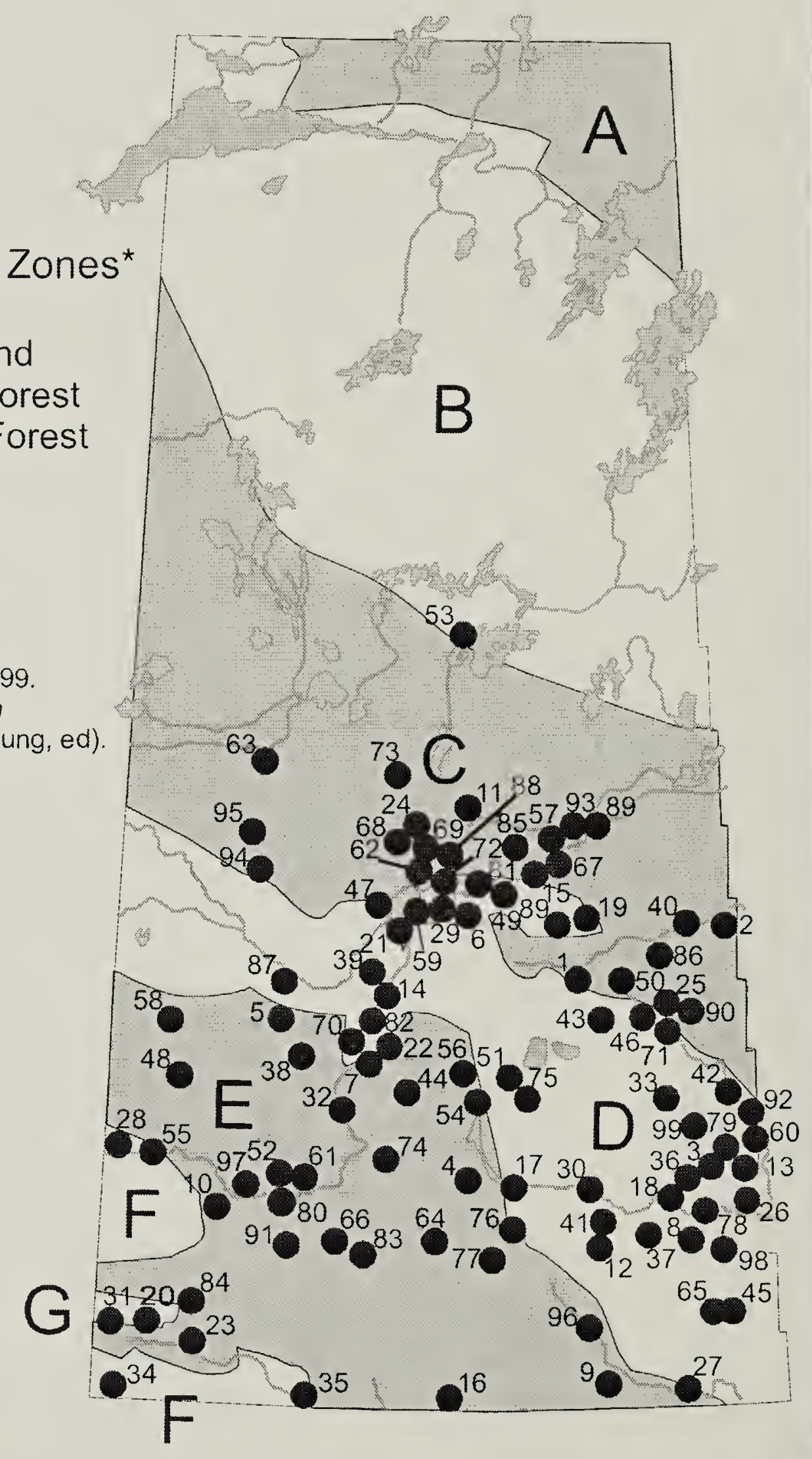
Aves

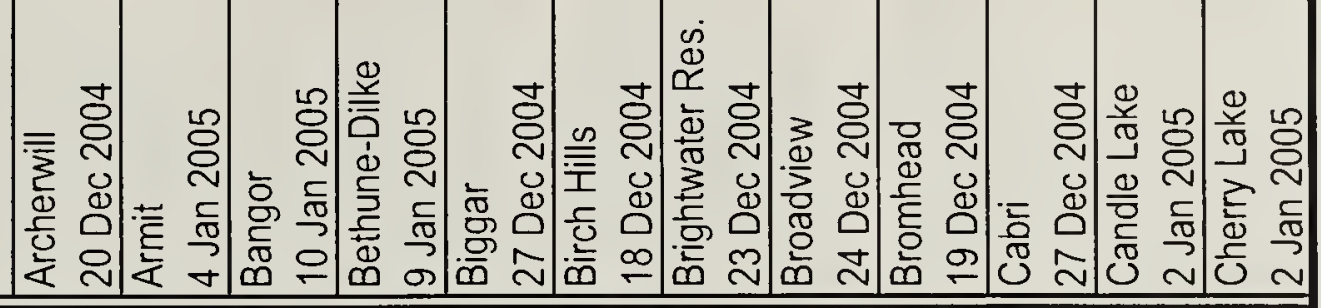

Canada Goose

Mallard

Common Goldeneye

Gray Partridge

Ring-necked Pheasant

Ruffed Grouse

Sharp-tailed Grouse

Bald Eagle

Northern Goshawk

Rough-legged Hawk

Golden Eagle

Merlin

Rock Pigeon

Great Horned Owl

Snowy Owl

Northern Hawk Owl

Great Gray Owl

Short-eared Owl

Downy Woodpecker

Hairy Woodpecker

Northern Flicker

Pileated Woodpecker

Northern Shrike

Gray Jay

Blue Jay

Black-billed Magpie

Common Raven

Horned Lark

Black-capped Chickadee

Boreal Chickadee

Red-breasted Nuthatch

White-breasted Nuthatch

Golden-crowned Kinglet

European Starling

Bohemian Waxwing

Cedar Waxwing

Dark-eyed Junco

Snow Bunting

Pine Grosbeak

House Finch

Common Redpoll

Hoary Redpoll

Pine Siskin

Evening Grosbeak

House Sparrow

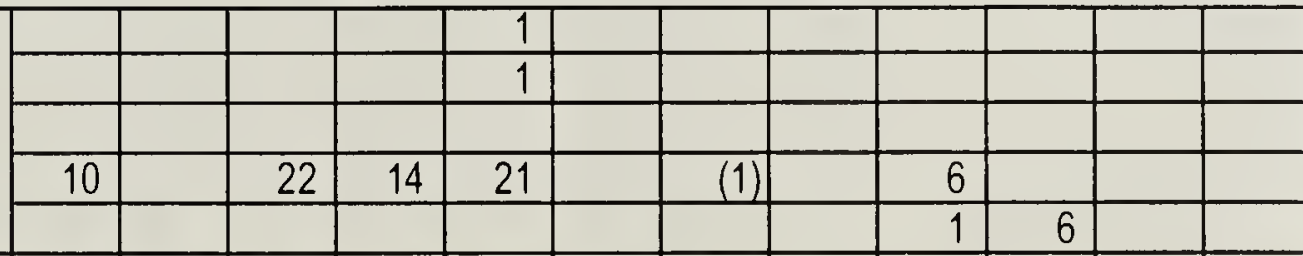

Total Birds Count Day

Total Birds Count Period

Total Species Count Day

Total Species Count Period

\begin{tabular}{|r|r|r|r|r|r|r|r|r|r|r|r|}
\hline 4 & 3 & & & & 4 & & 2 & & & & \\
\hline 3 & & 3 & 3 & 100 & & 5 & 30 & & 10 & & \\
\hline & & & & & & 1 & & & 1 & & \\
\hline & & & & & 1 & & & & & & \\
\hline & & & & & & & & & 2 & & \\
\hline & & & 1 & $(2)$ & & & & & 1 & & \\
\hline & & 20 & 16 & 96 & 59 & 18 & 19 & 17 & & & 16 \\
\hline$(1)$ & 1 & 4 & & 1 & 1 & 1 & 1 & & 3 & & \\
\hline & & & 1 & 1 & 2 & $(1)$ & & 2 & 2 & & \\
\hline$(1)$ & 1 & & & 1 & & & & & & & \\
\hline & 1 & & & & & & & & & 3 & \\
\hline & & & & & & & & & 3 & & \\
\hline 6 & 1 & 6 & & 6 & 7 & 1 & 3 & & & & 1 \\
\hline 6 & 1 & 4 & & 5 & 4 & & 1 & & & & \\
\hline & & & 2 & & & & & & & & \\
\hline 2 & & 1 & & & & & & & & & \\
\hline$(1)$ & & & & $(2)$ & $(1)$ & & 1 & & & 1 & \\
\hline 3 & 4 & & & & & & & & & 2 & \\
\hline 23 & 10 & & & $(1)$ & 6 & 3 & 1 & & & 2 & \\
\hline 4 & 4 & 33 & & 124 & 53 & 46 & 30 & & 14 & 13 & 3 \\
\hline 14 & 1 & 13 & & 27 & 15 & 5 & 10 & & & 151 & 2 \\
\hline$(15)$ & & & & 15 & 8 & & & 22 & 15 & & \\
\hline 44 & 14 & 18 & & 47 & 48 & 6 & 35 & & 7 & 43 & 7 \\
\hline 3 & & & & 2 & & & & & & 9 & \\
\hline 2 & 8 & & & 6 & & & $(1)$ & & & 1 & \\
\hline 3 & 1 & 6 & & & 1 & & 1 & & & & \\
\hline & & & & 4 & & & & & & & \\
\hline & & & & 1 & & & & 8 & & & \\
\hline & & & & 99 & 71 & 40 & 32 & & 80 & & \\
\hline & & & & & & & & & & & \\
\hline & 4 & 2 & & & & & & & & & \\
\hline$(30)$ & 8 & 100 & & 3416 & & 2 & 1 & 37 & 100 & & 76 \\
\hline 140 & 37 & & & 1 & 14 & 1 & & & & 3 & \\
\hline & & & & 11 & & & 3 & & & & \\
\hline 127 & 8 & 13 & & 67 & 257 & 12 & 42 & 1 & & 9 & \\
\hline & & & & 1 & & & & & & & \\
\hline & 2 & & & 7 & & & & & & & \\
\hline 88 & 68 & & & & & & & & & & \\
\hline 50 & & 75 & 6 & 673 & 77 & 152 & 115 & 211 & 25 & & \\
\hline 533 & 193 & 326 & 43 & 4735 & 629 & 293 & 340 & 334 & 270 & 238 & 105 \\
\hline 581 & 193 & 326 & 43 & 4742 & 630 & 295 & 341 & 334 & 272 & 238 & 105 \\
\hline 19 & 22 & 16 & 7 & 27 & 18 & 14 & 19 & 11 & 15 & 12 & 6 \\
\hline 24 & 22 & 16 & 7 & 31 & 19 & 16 & 20 & 11 & 16 & 12 & 6 \\
\hline
\end{tabular}


Aves

Canada Goose

Mallard

Common Goldeneye

Gray Partridge

Ring-necked Pheasant

Ruffed Grouse

Sharp-tailed Grouse

Bald Eagle

Northern Goshawk

Rough-legged Hawk

Golden Eagle

Merlin

Rock Pigeon

Great Horned Owl

Snowy Owl

Northern Hawk Owl

Great Gray Owl

Short-eared Owl

Downy Woodpecker

Hairy Woodpecker

Northern Flicker

Pileated Woodpecker

Northern Shrike

Gray Jay

Blue Jay

Black-billed Magpie

Common Raven

Horned Lark

Black-capped Chickadee

Boreal Chickadee

Red-breasted Nuthatch

White-breasted Nuthatch

Golden-crowned Kinglet

European Starling

Bohemian Waxwing

Cedar Waxwing

Dark-eyed Junco

Snow Bunting

Pine Grosbeak

House Finch

Common Redpoll

Hoary Redpoll

Pine Siskin

Evening Grosbeak

Housc Soarrow

Total Birds Count Day

Total Birds Count Period

Total Species Count Day

Total Species Count Period

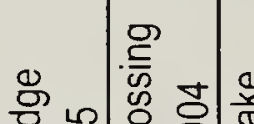

은

它

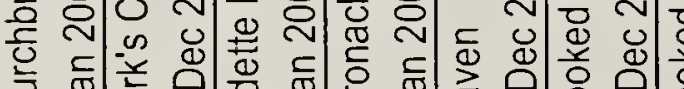

䨔

U v

\begin{tabular}{|r|r|r|r|r|r|r|r|r|r|r|r|}
\hline & 1361 & & 1150 & & 4634 & & & & & & \\
\hline & & & 26017 & & 89 & & & & & 11 & \\
\hline & 45 & & & & 3 & & & & & & \\
\hline & 57 & 11 & 38 & 9 & & & & & 24 & 19 & \\
\hline & & & & & & & 1 & & & 7 & \\
\hline & 20 & 2 & 24 & 17 & & & 3 & 3 & & 2 & \\
\hline & 1 & 1 & & 1 & 3 & & 1 & & & 1 & \\
\hline & & & & & & & & 2 & & & \\
\hline & 1 & & & & & & & & & & \\
\hline & & & & 2 & 1 & & 1 & & & 3 & \\
\hline & 1 & & & & & & & & & & \\
\hline & 119 & & 81 & 201 & 44 & & & 55 & 1 & 10 & \\
\hline & 11 & & 3 & & & & & & 2 & & \\
\hline & 3 & & 3 & 1 & & & & & 1 & & \\
\hline
\end{tabular}

\begin{tabular}{|c|c|c|c|c|c|c|c|c|c|c|c|}
\hline & & & & & & & & & & & \\
\hline & & 1 & & & & & & & & & \\
\hline & 1 & & & & & & & & & & \\
\hline 5 & 3 & & 1 & 8 & 1 & 2 & 2 & & 1 & 10 & 2 \\
\hline 6 & 10 & 3 & & 8 & 3 & 2 & 5 & 2 & (1) & 2 & 2 \\
\hline
\end{tabular}

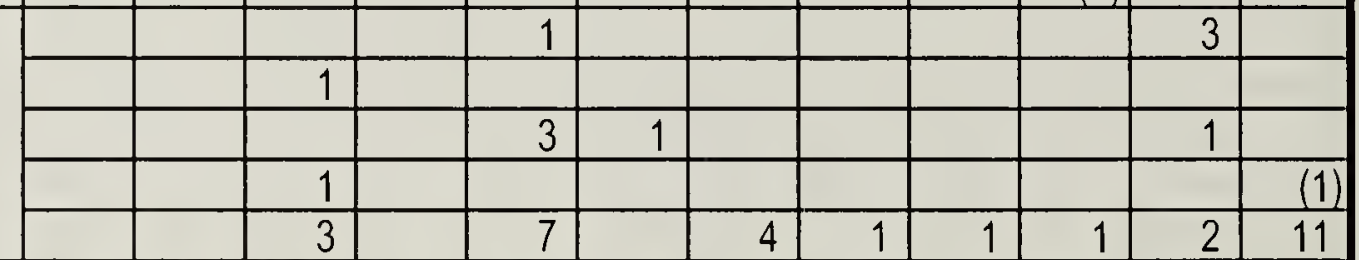

\begin{tabular}{|r|r|r|r|r|r|r|r|r|r|r|r|}
\hline 3 & 383 & 17 & 1 & 85 & 18 & 3 & 16 & 18 & 58 & 34 & \\
\hline 3 & 31 & 26 & & 19 & 17 & 5 & 2 & 30 & 7 & & 104 \\
\hline & 1 & & 384 & & 1 & & & & 19 & 5 & \\
\hline 45 & 119 & 12 & & 86 & 51 & 9 & 49 & 18 & 21 & 26 & 117 \\
\hline
\end{tabular}

\begin{tabular}{|r|r|r|r|r|r|r|r|r|r|r|r|}
\hline & 3 & & & 2 & & & 17 & & & 2 & 9 \\
\hline 2 & & & & 11 & 2 & & 1 & & & & 4 \\
\hline & & & & 7 & & & 11 & & & 1 & \\
\hline
\end{tabular}

\begin{tabular}{|r|r|r|r|r|l|r|r|r|r|r|r|}
\hline & 15 & & 5 & 32 & & & 3 & & 1 & & \\
\hline 10 & 406 & & 7 & 58 & & & 2 & & 4 & 45 & \\
\hline & & & & & & & 24 & & & 6 & \\
\hline 1 & & & & & & & & & & 20 & \\
\hline & 35 & & 45 & 1 & & 74 & 25 & 25 & 457 & & \\
\hline & 10 & & & & & 8 & 1 & 13 & & 2 & 10 \\
\hline & 33 & & 4 & 7 & & & & & & 60 & \\
\hline 25 & 67 & 100 & 1 & 80 & 50 & 14 & 48 & 60 & & 131 & 92 \\
\hline & & & & & & 15 & & & & & \\
\hline & & 7 & & $(2)$ & & & 18 & & & & \\
\hline & & 8 & & & & 10 & & & & & 36 \\
\hline 30 & 2254 & 1 & 180 & 608 & 66 & & 10 & 95 & 28 & 14 & 30 \\
\hline
\end{tabular}

\begin{tabular}{|r|r|r|r|r|r|r|r|r|r|r|r|}
\hline \hline 130 & 4993 & 236 & 28012 & 1264 & 4989 & 146 & 266 & 332 & 625 & 456 & 420 \\
\hline
\end{tabular}

\begin{tabular}{|r|r|r|r|r|r|r|r|r|r|r|r|}
130 & 4993 & 236 & 28012 & 1267 & 4989 & 146 & 266 & 332 & 626 & 457 & 423 \\
\hline
\end{tabular}

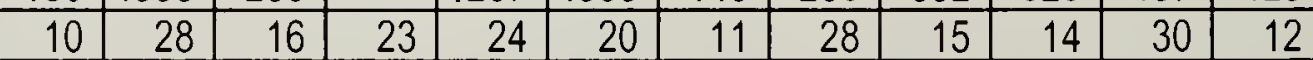

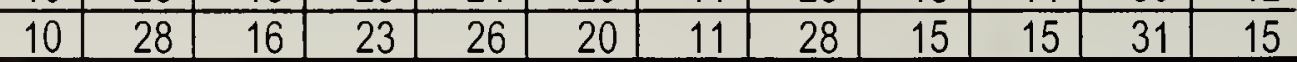


Table 3-3 Species found in 9 or more localities ()$=$ seen during count period

Aves

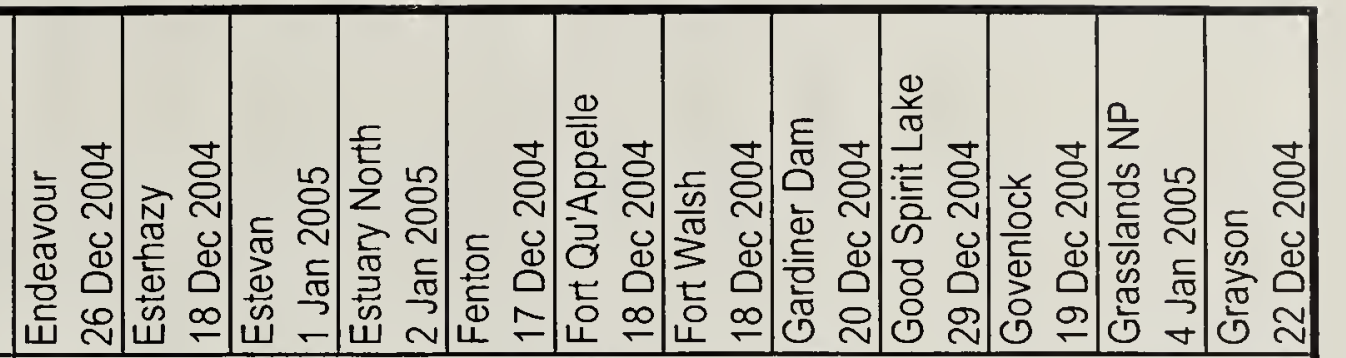

Canada Goose

Mallard

Common Goldeneye

Gray Partridge

Ring-necked Pheasant

Ruffed Grouse

Sharp-tailed Grouse

Bald Eagle

Northern Goshawk

Rough-legged Hawk

Golden Eagle

Merlin

Rock Pigeon

Great Horned Owl

Snowy Owl

Northern Hawk Owl

Great Gray Owl

Short-eared Owl

Downy Woodpecker

Hairy Woodpecker

Northern Flicker

Pileated Woodpecker

Northern Shrike

Gray Jay

Blue Jay

Black-billed Magpie

Common Raven

Horned Lark

Black-capped Chickadee

Boreal Chickadee

Red-breasted Nuthatch

White-breasted Nuthatch

Golden-crowned Kinglet

European Starling

Bohemian Waxwing

Cedar Waxwing

Dark-eyed Junco

Snow Bunting

Pine Grosbeak

House Finch

Common Redpoll

Hoary Redpoll

Pine Siskin

Evening Grosbeak

House Sparrow

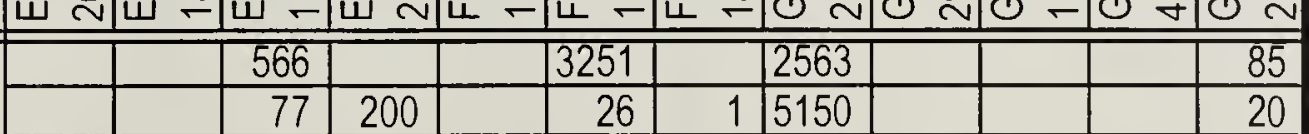

Total Birds Count Day

\begin{tabular}{|r|r|r|r|r|r|r|r|r|r|r|r|}
\hline & & 6 & & & 5 & & 62 & & & & \\
\hline & 4 & & 18 & 23 & & 21 & 61 & & 32 & 24 & \\
\hline & & 3 & 20 & & & 4 & & & 7 & & \\
\hline 1 & & & & 1 & $(1)$ & 2 & & 3 & & & 1 \\
\hline & 12 & & 50 & & & 10 & 6 & 1 & & 20 & 9 \\
\hline & & & & & 1 & 5 & 17 & $(1)$ & 1 & & \\
\hline & & & 3 & $(1)$ & & 2 & & & & & \\
\hline
\end{tabular}

Total Birds Count Period

Total Species Count Day

Total Species Count Period

\begin{tabular}{|c|c|c|c|c|c|c|c|c|c|c|c|}
\hline & & & & & & & & & & & \\
\hline (1) & (1) & & $\underline{5}$ & 6 & 7 & & 3 & 19 & & & 11 \\
\hline 7 & 10 & 1 & 42 & 35 & 29 & 86 & 81 & 26 & 28 & 20 & 31 \\
\hline 50 & $\overline{4}$ & 1 & & 21 & 12 & & 3 & 27 & 1 & & 29 \\
\hline & & 3 & 50 & 4 & & 3 & 18 & 2 & 86 & 300 & \\
\hline 12 & 9 & $\overline{4}$ & 10 & 48 & 38 & 59 & 13 & 88 & & & 39 \\
\hline & & & & 1 & & & & & & & \\
\hline & 1 & $(1)$ & & & 2 & 4 & 1 & 1 & & & 2 \\
\hline 2 & & 1 & & 2 & 10 & & & 2 & & & (1) \\
\hline & & & & 2 & & 1 & 1 & & & & \\
\hline & & 2 & & & & 7 & & & 4 & & 3 \\
\hline & & 54 & 400 & 51 & 60 & 36 & & 250 & & & 8 \\
\hline & & & & & & & & & & & \\
\hline & & & 1 & & & 8 & 1 & & & & \\
\hline 50 & & 37 & 126 & & 15 & 420 & 1311 & 52 & 28 & 359 & \\
\hline 16 & & & $\overline{9}$ & & $(5$ & 12 & 6 & 12 & & & \\
\hline & (1) & 3 & & & 7 & & 1 & & & & \\
\hline 8 & 35 & 15 & & 128 & 9 & 81 & 33 & 63 & 22 & 16 & 602 \\
\hline & & & & & & 2 & & & & & \\
\hline & & & & & 4 & & & & & & \\
\hline 2 & & & & & & & & & & & \\
\hline (1) & 21 & 121 & 8 & 30 & 117 & 190 & 528 & 72 & 136 & & 9 \\
\hline 160 & 100 & 945 & 970 & 358 & 3686 & 1017 & 10022 & 636 & $\overline{355}$ & 812 & $\overline{984}$ \\
\hline 162 & 102 & 961 & 970 & 360 & 3708 & 1017 & 10022 & 637 & 355 & 812 & 985 \\
\hline 14 & 10 & 20 & 20 & 16 & 22 & 37 & 35 & 19 & 17 & 12 & 17 \\
\hline 16 & 12 & 24 & 20 & 18 & 31 & 37 & 35 & 20 & 17 & 12 & 18 \\
\hline
\end{tabular}


Table 3-4 Species found in 9 or more localities () = seen during count period

Aves

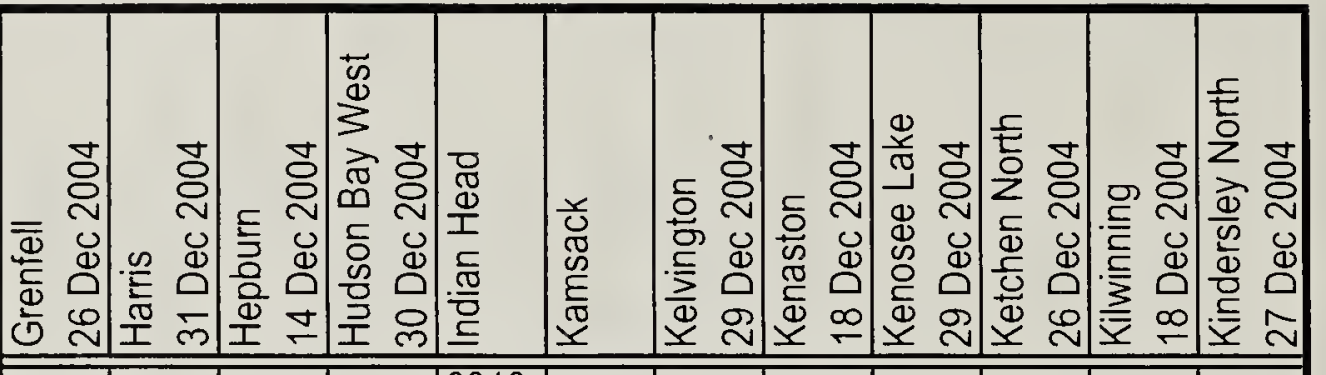

Canada Goose

Mallard

Common Goldeneye

Gray Partridge

Ring-necked Pheasant

Ruffed Grouse

Sharp-tailed Grouse

Bald Eagle

Northern Goshawk

Rough-legged Hawk

Golden Eagle

Merlin

Rock Pigeon

Great Horned Owl

Snowy Owl

Northern Hawk Owl

Great Gray Owl

Short-eared Owl

Downy Woodpecker

Hairy Woodpecker

Northern Flicker

Pileated Woodpecker

Northern Shrike

Gray Jay

Blue Jay

Black-billed Magpie

Common Raven

Horned Lark

Black-capped Chickadee

Boreal Chickadee

Red-breasted Nuthatch

White-breasted Nuthatch

Golden-crowned Kinglet

European Starling

Bohemian Waxwing

Cedar Waxwing

Dark-eyed Junco

Snow Bunting

Pine Grosbeak

House Finch

Common Redpoll

Hoary Redpoll

Pine Siskin

Evening Grosbeak

House Sparrow

\begin{tabular}{|r|r|r|r|r|r|r|r|r|r|r|r|}
\hline & & & & 150 & & & & & & & \\
\hline & & & & 1 & & & & & & & \\
\hline & 32 & & & 89 & 6 & & 11 & & & 1 & 27 \\
\hline
\end{tabular}

Total Birds Count Day

Total Birds Count Period

Total Species Count Day

Total Species Count Period

\begin{tabular}{|r|r|r|r|r|r|r|r|r|r|r|r|}
\hline & & & & & & & & & & & 27 \\
\hline & 4 & & 6 & 164 & & & & & 5 & & \\
\hline & & & $(1)$ & 22 & & & & & & 6 & \\
\hline & & & & & 3 & & & & & & \\
\hline
\end{tabular}

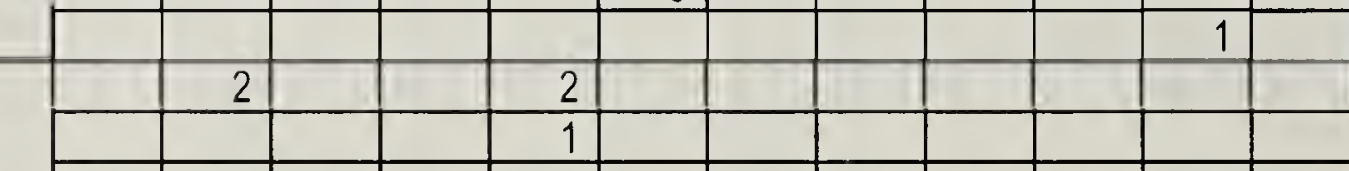

\begin{tabular}{|r|r|r|r|r|r|r|r|r|r|r|r|}
\hline 3 & 149 & & & 50 & & 2 & 12 & & 4 & 9 & 6 \\
\hline & & & 1 & 3 & & & & & 1 & 2 & 4 \\
\hline & & & & 8 & 2 & & 1 & & & & 2 \\
\hline
\end{tabular}


Table 3-5 Species found in 9 or more localities ()$=$ seen during count period

Aves

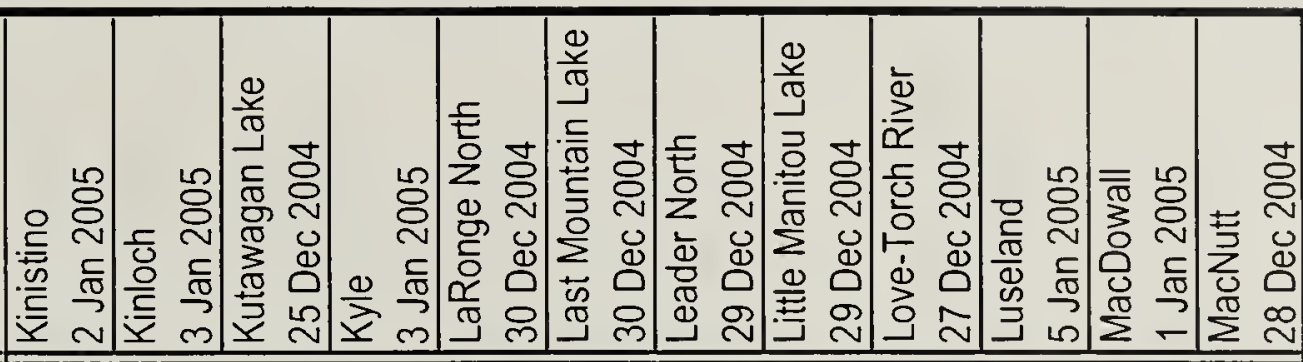

Canada Goose

Mallard

Common Goldeneye

Gray Partridge

Ring-necked Pheasant

Ruffed Grouse

Sharp-tailed Grouse

Bald Eagle

Northern Goshawk

Rough-legged Hawk

Golden Eagle

Merlin

Rock Pigeon

Great Horned Owl

Snowy Owl

Northern Hawk Owl

Great Gray Owl

Short-eared Owl

Downy Woodpecker

Hairy Woodpecker

Northern Flicker

Pileated Woodpecker

Northern Shrike

Gray Jay

Blue Jay

Black-billed Magpie

Common Raven

Horned Lark

Black-capped Chickadee

Boreal Chickadee

Red-breasted Nuthatch

White-breasted Nuthatch

Golden-crowned Kinglet

European Starling

Bohemian Waxwing

Cedar Waxwing

Dark-eyed Junco

Snow Bunting

Pine Grosbeak

House Finch

Common Redpoll

Hoary Redpoll

Pine Siskin

Evening Grosbeak

House Sparrow

\begin{tabular}{|l|l|l|l|l|l|l|l|l|l|l|l|}
\hline & & & & & & & & & & & \\
\hline & & & & & & & & & & & \\
\hline & & 22 & 224 & & 36 & 15 & 8 & 30 & 26 & $(15)$ & 21 \\
\hline & & & & & & 35 & & & & & \\
\hline
\end{tabular}

(n)

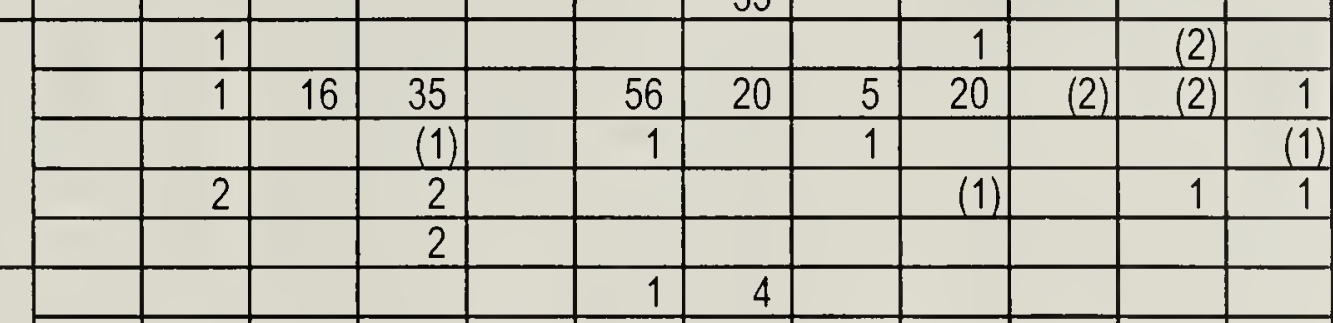

Total Birds Count Day

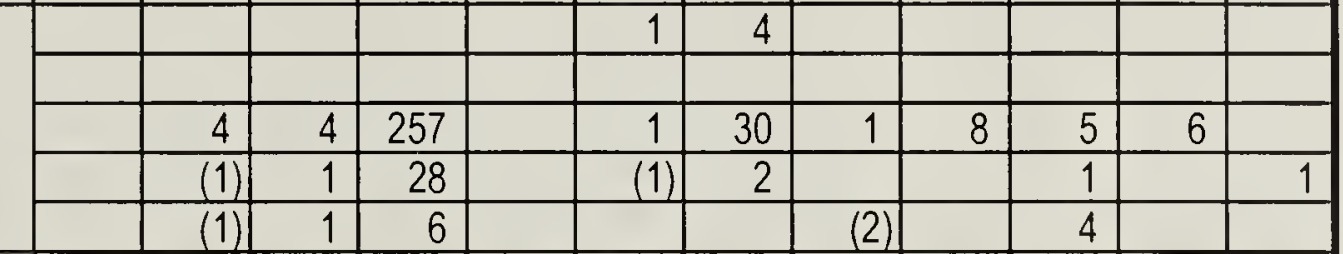

Total Birds Count Period

Total Species Count Day

Total Species Count Period 
Table 3-6 Species found in 9 or more localities ()$=$ seen during count period

Aves

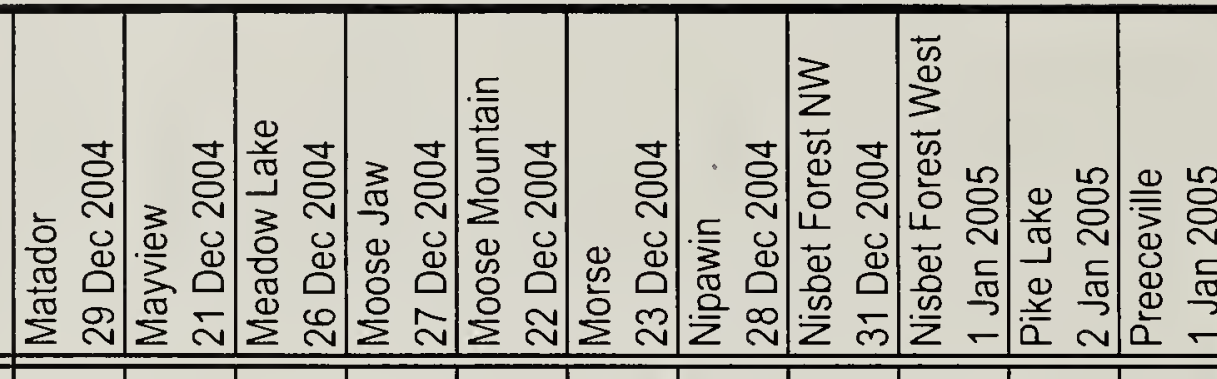

Canada Goose

Mallard

Common Goldeneye

Gray Partridge

Ring-necked Pheasant

Ruffed Grouse

Sharp-tailed Grouse

Bald Eagle

Northern Goshawk

Rough-legged Hawk

Golden Eagle

Merlin

Rock Pigeon

Great Horned Owl

Snowy Owl

Northern Hawk Owl

Great Gray Owl

Short-eared Owl

Downy Woodpecker

Hairy Woodpockor

Northern Flicker

Pileated Woodpecker

Northern Shrike

Gray Jay

Blue Jay

Black-billed Magpie

Common Raven

Horned Lark

Black-capped Chickadee

Boreal Chickadee

Red-breasted Nuthatch

White-breasted Nuthatch

Golden-crowned Kinglet

European Starling

Bohemian Waxwing

Cedar Waxwing

Dark-eyed Junco

Snow Bunting

Pine Grosbeak

House Finch

Common Redpoll

Hoary Redpoll

Pine Siskin

Evening Grosbeak

House Sparrow

\begin{tabular}{|r|r|r|r|r|r|r|r|r|r|r|}
\hline & & & & & & & & & & \\
\hline & & & & & & & & & & \\
\hline & & 12 & 25 & & 60 & 12 & & $(8)$ & 14 & \\
\hline & & & 1 & & & & & & & \\
\hline$(5)$ & 3 & 2 & & & & 3 & $(4)$ & 1 & & \\
\hline & & & & & 54 & 7 & & 1 & 114 & \\
\hline & & & & & & 1 & & 1 & & \\
\hline 1 & & & & & $(1)$ & & & & & \\
\hline 1 & & & & & & & & & & \\
\hline
\end{tabular}

Total Birds Count Day

Total Birds Count Period

Total Species Count Day

Total Species Count Period

\begin{tabular}{|c|c|c|c|c|c|c|c|c|c|c|}
\hline & & & 268 & 34 & 32 & & (12) & & 84 & \\
\hline & & & 4 & & 7 & 1 & & & 3 & \\
\hline & & & 4 & & 1 & & & 1 & & \\
\hline & & $(1)$ & & & & 1 & & 4 & & \\
\hline & & & & & & 1 & (1) & 1 & & \\
\hline & & & & & & & (1) & & & \\
\hline & & 5 & 6 & 2 & & 6 & 2 & 6 & 28 & $\overline{6}$ \\
\hline & & 3 & 3 & 9 & & 7 & 2 & 8 & 27 & 6 \\
\hline & & & & & & & & & 2 & \\
\hline & 1 & & & & & (1) & & & 1 & 3 \\
\hline & & & & & & & & & 3 & \\
\hline & 2 & 5 & & & & 3 & 1 & 2 & & \\
\hline & 4 & 4 & 1 & 5 & & 12 & 7 & 19 & 37 & 6 \\
\hline 3 & & 2 & 32 & 16 & 16 & 34 & (2) & 15 & 284 & 8 \\
\hline & 2 & 21 & & 8 & & 78 & 4 & 13 & 54 & 1 \\
\hline & & & 3 & & 89 & & & & 18 & \\
\hline & 6 & 35 & 25 & 31 & & 41 & 24 & 43 & 416 & 50 \\
\hline & 1 & & & & & & (1) & & - & \\
\hline & & 3 & 21 & 1 & & 8 & 2 & & 4 & 1 \\
\hline & & 3 & 2 & & & 4 & 3 & 4 & 11 & 2 \\
\hline & & & & 3 & & & & & & \\
\hline & & & 10 & & & & & & 13 & \\
\hline 6 & & & & & $\overline{(50)}$ & 70 & 45 & 19 & 65 & \\
\hline & & & & & & & & & & \\
\hline & & & 12 & & & & & & & \\
\hline$(200)$ & & 1000 & 70 & & 130 & & (55) & 875 & 1350 & \\
\hline & 6 & 29 & 1 & 8 & & 106 & 4 & 46 & 176 & 47 \\
\hline & & & 63 & & 6 & & & & 1 & \\
\hline & 2 & 68 & & & 50 & 43 & 14 & 600 & 506 & 75 \\
\hline & & & & & & & & 24 & 1 & \\
\hline & & & 13 & & 2 & 30 & 4 & & 2 & \\
\hline & 4 & 8 & & & & 125 & (6) & 87 & 1 & 12 \\
\hline & & & 272 & 156 & 745 & 98 & (13) & & 492 & \\
\hline$\overline{\overline{12}}$ & $\overline{31}$ & 11201 & 855 & 273 & 1193 & 2691 & 112 & $\overline{17770}$ & 3713 & $\overline{2217}$ \\
\hline 217 & 32 & 1203 & 855 & 273 & 1244 & 692 & 208 & 1778 & 3713 & 217 \\
\hline 5 & 10 & 16 & 22 & 11 & 13 & 22 & 12 & 20 & 30 & 12 \\
\hline 7 & 11 & 18 & 22 & 11 & 15 & 23 & 22 & 21 & 30 & 12 \\
\hline
\end{tabular}


Table 3-7 Species found in 9 or more localities ()$=$ seen during count period

Aves

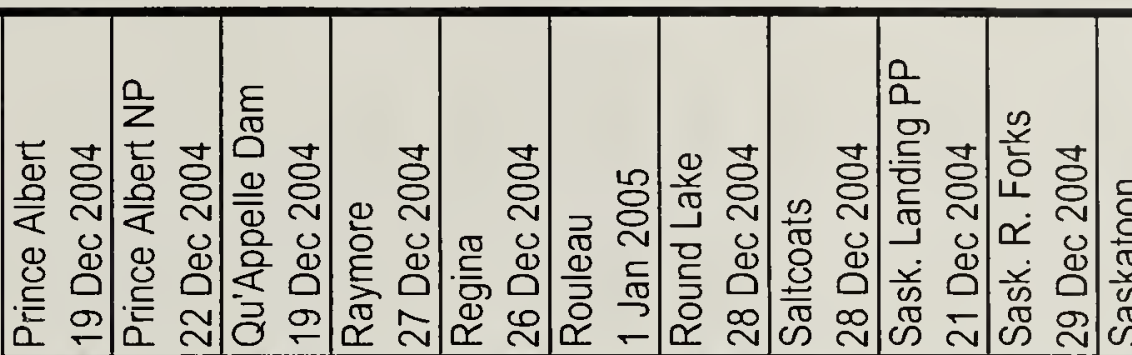

Canada Goose

Mallard

Common Goldeneye

Gray Partridge

Ring-necked Pheasant

Ruffed Grouse

Sharp-tailed Grouse

Bald Eagle

Northern Goshawk

Rough-legged Hawk

Golden Eagle

Merlin

Rock Pigeon

Great Horned Owl

Snowy Owl

Northern Hawk Owl

Great Gray Owl

Short-eared Owl

Downy Woodpecker

Hairy Woodpecker

Northern Flicker

Pileated Woodpecker

Northern Shrike

Gray Jay

Blue Jay

Black-billed Magpie

Common Raven

Horned Lark

Black-capped Chickadee

Boreal Chickadee

Red-breasted Nuthatch

White-breasted Nuthatch

Golden-crowned Kinglet

European Starling

Bohemian Waxwing

Cedar Waxwing

Dark-eyed Junco

Snow Bunting

Pine Grosbeak

House Finch

Common Redpoll

Hoary Redpoll

Pine Siskin

Evening Grosbeak

House Sparrow.

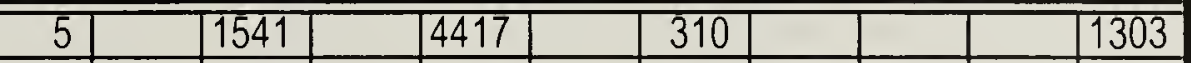

Total Birds Count Day

Total Birds Count Period

Total Species Count Day

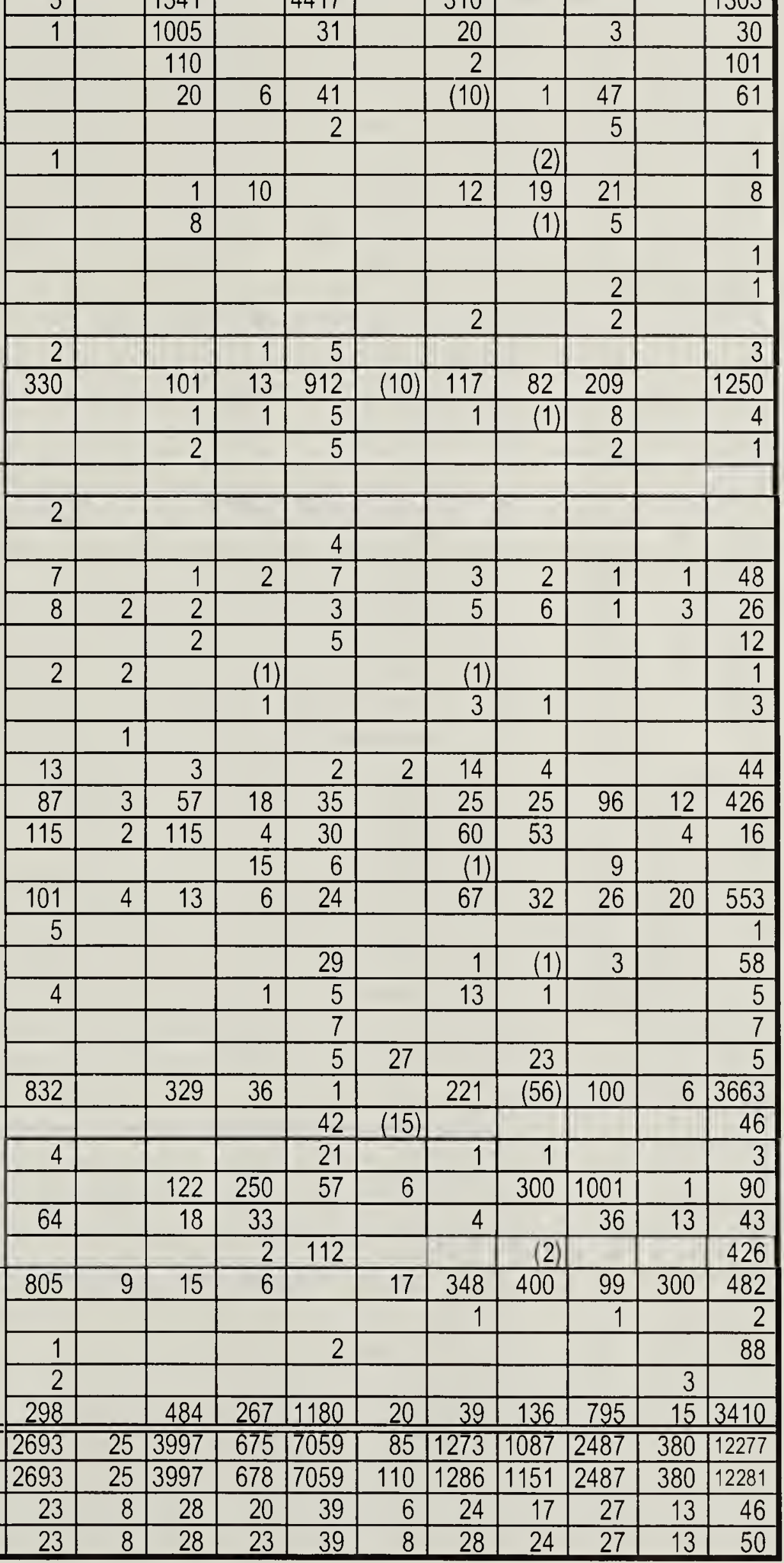


Table 3-8 Species found in 9 or more localities $\quad()=$ seen during count period

Aves

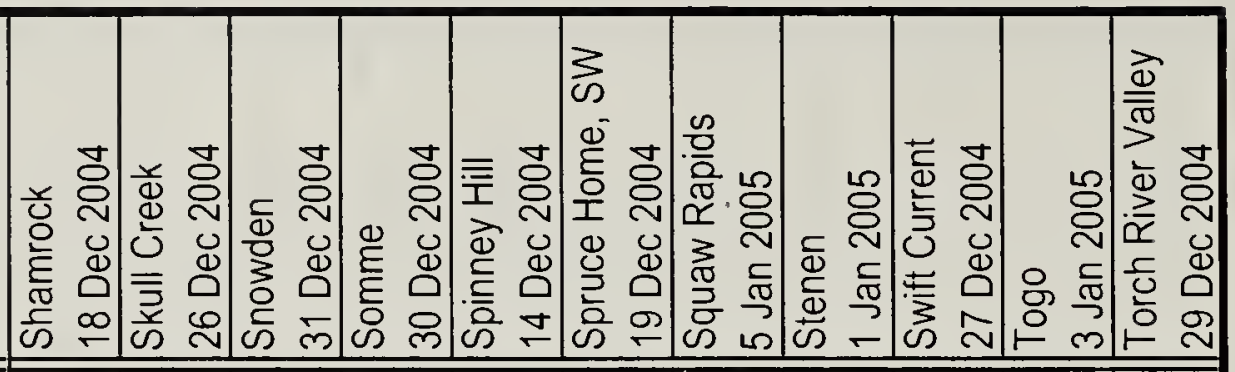

Canada Goose

Mallard

Common Goldeneye

Gray Partridge

Ring-necked Pheasant

Ruffed Grouse

Sharp-tailed Grouse

Bald Eagle

Northern Goshawk

Rough-legged Hawk

Golden Eagle

Merlin

Rock Pigeon

Great Horned Owl

Snowy Owl

Northern Hawk Owl

Great Gray Owl

Short-eared Owl

Downy Woodpecker

Hairy Woodpecker

Northern Flicker

Pileated Woodpecker

Northern Shrike

Gray Jay

Blue Jay

Black-billed Magpie

Common Raven

Horned Lark

Black-capped Chickadee

Boreal Chickadee

Red-breasted Nuthatch

White-breasted Nuthatch

Golden-crowned Kinglet

European Starling

Bohemian Waxwing

Cedar Waxwing

Dark-eyed Junco

Snow Bunting

Pine Grosbeak

House Finch

Common Redpoll

Hoary Redpoll

Pine Siskin

Evening Grosbeak

Housc Soarrow

\begin{tabular}{|l|l|l|l|}
\hline & & & \\
\hline & & & \\
\hline & & & \\
\hline 41 & & 17 &
\end{tabular}

Total Birds Count Day

Total Birds Count Period

Total Species Count Day

Total Species Count Period

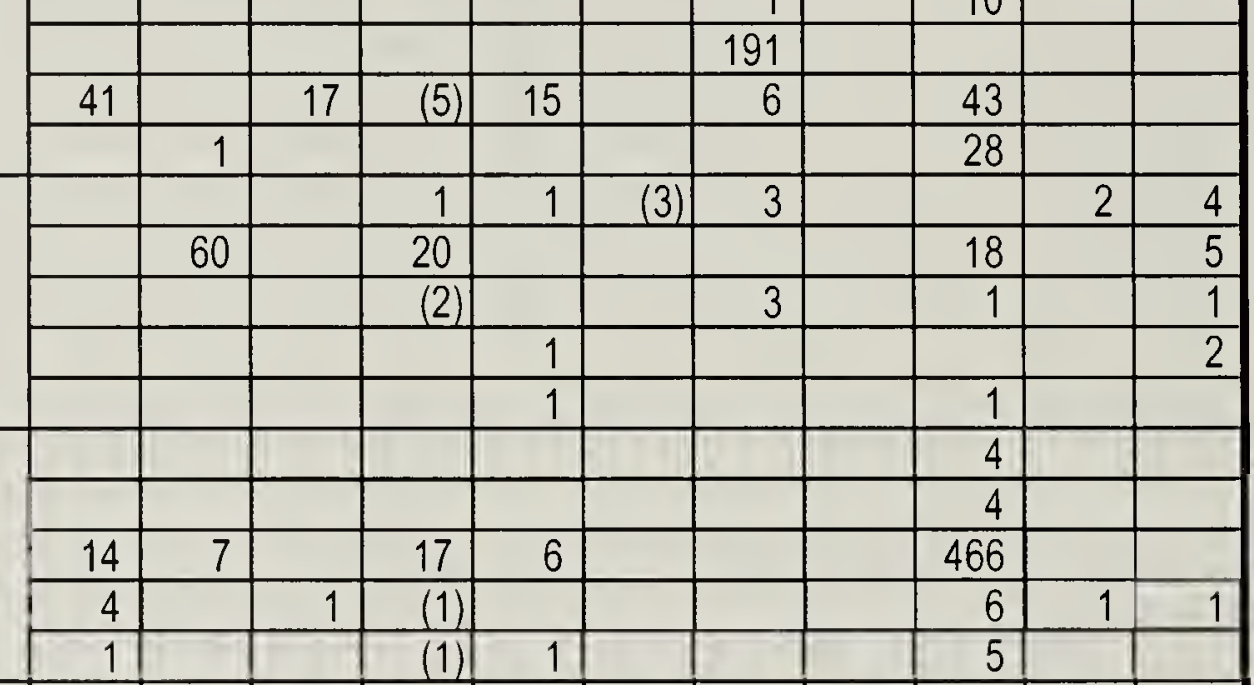

\begin{tabular}{|r|r|r|r|r|r|r|r|r|r|r|}
\hline & & 1 & & & & 3 & & & & \\
\hline & & 2 & $(1)$ & & & 3 & & & & 1 \\
\hline & & & & & & & & & & \\
\hline & 3 & 5 & 7 & 2 & 2 & 1 & 2 & 7 & 6 & 6 \\
\hline
\end{tabular}


Table 3-9 Species found in 9 or more localities ()$=$ seen during count period

Aves

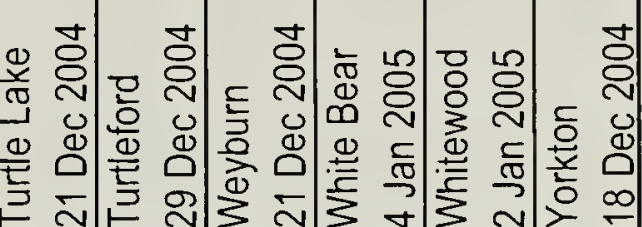

Canada Goose

Mallard

Common Goldeneye

Gray Partridge

Ring-necked Pheasant

Ruffed Grouse

Sharp-tailed Grouse

Bald Eagle

Northern Goshawk

Rough-legged Hawk

Golden Eagle

Merlin

Rock Pigeon

Great Horned Owl

Snowy Owl

Northern Hawk Owl

Great Gray Owl

Short-eared Owl

Downy Woodpecker

Hairy Woodpecker

Northern Flicker

Pileated Woodpecker

Northern Shrike

Gray Jay

Blue Jay

Black-billed Magpie

Common Raven

Horned Lark

Black-capped Chickadee

Boreal Chickadee

Red-breasted Nuthatch

White-breasted Nuthatch

Golden-crowned Kinglet

European Starling

Bohemian Waxwing

Cedar Waxwing

Dark-eyed Junco

Snow Bunting

Pine Grosbeak

House Finch

Common Redpoll

Hoary Redpoll

Pine Siskin

Evening Grosbeak

House Sparrow

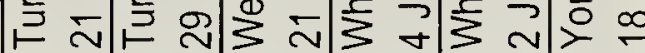

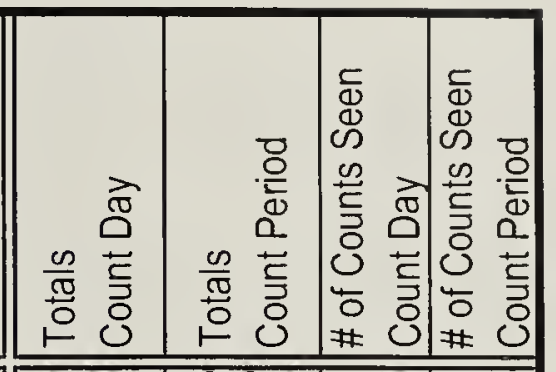

Total Birds Count Day

Total Birds Count Period

\begin{tabular}{|l|l|l|l|l|l|}
\hline & 6 & & & & \\
\hline & & & & & 185 \\
\hline
\end{tabular}

\begin{tabular}{|r|r|r|r|}
\hline 24203 & 24243 & 15 & 16 \\
\hline 33028 & 33028 & 20 & 20 \\
\hline 526 & 526 & 10 & 10 \\
\hline
\end{tabular}

Total Species Count Day

\begin{tabular}{|r|r|r|r|r|r||}
\hline 15 & 30 & 12 & 52 & $(1)$ & 24 \\
\hline & & 3 & & & \\
\hline 6 & 1 & & & 1 & \\
\hline 15 & 5 & 6 & 6 & 7 & \\
\hline 2 & & 1 & & & $(1)$ \\
\hline
\end{tabular}

\begin{tabular}{|r|r|r|r|}
\hline 526 & 526 & 10 & 10 \\
\hline 1516 & 1556 & 53 & 59 \\
\hline
\end{tabular}

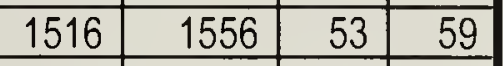

\begin{tabular}{ll|l}
61 & 73 & 28 \\
\hline
\end{tabular}

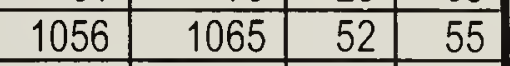

Total Species Count Period

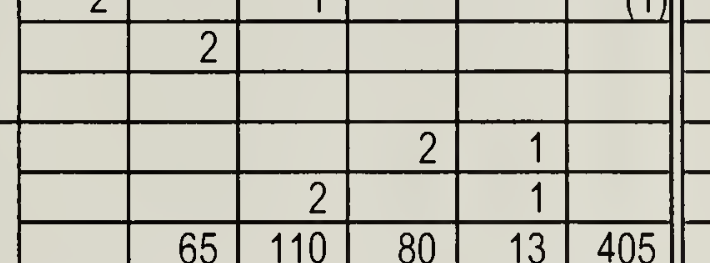

\begin{tabular}{l|r|r|r|}
\hline 79 & 88 & 22 & 30 \\
\hline 25 & 27 & 15 & 17 \\
\hline 19 & 20 & 13 & 14 \\
\hline
\end{tabular}

\begin{tabular}{|r|r|r|r|}
\hline 19 & 20 & 13 & 14 \\
\hline 36 & 39 & 20 & 22 \\
\hline 21 & 22 & 10 & 11 \\
\hline 6247 & 6269 & 63 & 65 \\
\hline
\end{tabular}

\begin{tabular}{|r|r|r|r|}
\hline 39 & 146 & 45 & 52 \\
\hline 77 & 84 & 30 & 35 \\
\hline
\end{tabular}

\begin{tabular}{|l|l|l|l|}
\hline 16 & 18 & 11 & 13 \\
\hline 21 & 30 & 12 & 15 \\
\hline
\end{tabular}

\begin{tabular}{|l|r|r|r|}
\hline 21 & 30 & 12 & 15 \\
\hline
\end{tabular}

\begin{tabular}{|r|r|r|r|}
53 & 54 & 9 & 10 \\
\hline 389 & 390 & 77 & 78 \\
\hline
\end{tabular}

\begin{tabular}{|r|r|r|r|}
\hline 389 & 390 & 77 & 78 \\
\hline 374 & 378 & 71 & 75 \\
\hline 36 & 36 & 11 & 11 \\
\hline
\end{tabular}

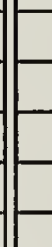

\begin{tabular}{|l|r|r|r|}
36 & 36 & 11 & 11 \\
\hline 38 & 46 & 23 & 30 \\
\hline
\end{tabular}

\begin{tabular}{|l|l|l|r|}
\hline 38 & 46 & 23 & 30 \\
\hline 33 & 41 & 23 & 30 \\
\hline
\end{tabular}

\begin{tabular}{|r|r|r|r|}
\hline 84 & 85 & 22 & 23 \\
\hline 733 & 736 & 67 & 70 \\
\hline 3861 & 3869 & 90 & 92 \\
\hline
\end{tabular}

\begin{tabular}{|r|r|r|r|r|r|}
\hline 10 & 4 & 3 & & 1 & 5 \\
\hline 2 & 17 & 7 & 63 & 61 & 62 \\
\hline 5 & 40 & & & 12 & 47 \\
\hline
\end{tabular}

\begin{tabular}{|r|r|r|r|}
\hline 3861 & 3869 & 90 & 92 \\
\hline 2614 & 2619 & 75 & 77 \\
\hline 1449 & 1466 & 37 & 40 \\
\hline 4295 & 4295 & 87 & 87 \\
\hline 58 & 59 & 18 & 19 \\
\hline
\end{tabular}

\begin{tabular}{r|r|r|r|r|r||}
\hline 15 & 38 & 2 & & 161 & 55 \\
\hline 3 & & & & & \\
\hline
\end{tabular}

\begin{tabular}{|r|r|r|r|}
\hline 4295 & 4295 & 87 & 87 \\
\hline 357 & 362 & 18 & 19 \\
\hline 247 & 248 & 46 & 46 \\
\hline 55 & 57 & 12 & 13 \\
\hline
\end{tabular}

\begin{tabular}{|r|r|r|r|r|r|}
\hline 2 & & 3 & & 1 & 4 \\
\hline 3 & 1 & 4 & & 15 & 4 \\
\hline & & & & & $(2)$ \\
\hline & & 1 & 30 & 1 & \\
\hline
\end{tabular}

\begin{tabular}{|r|r|r|r|}
\hline 247 & 248 & 46 & 47 \\
\hline 55 & 57 & 12 & 13 \\
\hline 223 & 223 & 24 & 24 \\
\hline 7615 & 7723 & 45 & 49 \\
\hline 681 & 716 & 10 & 12 \\
\hline 189 & 190 & 23 & 24 \\
\hline 25216 & 25506 & 67 & 71 \\
\hline 2229 & 2234 & 59 & 60 \\
\hline 1293 & 1296 & 23 & 25 \\
\hline 9541 & 9541 & 84 & 84 \\
\hline 85 & 85 & 16 & 16 \\
\hline 324 & 326 & 20 & 21 \\
\hline 1701 & 1713 & 32 & 34 \\
\hline 23098 & 23112 & 78 & 80 \\
\hline 155042 & & & \\
\hline
\end{tabular}

\begin{tabular}{r|r|r|r|r|r|}
\hline 7 & & & & \\
\hline
\end{tabular}

\begin{tabular}{l|l|l|l|l|l|l|l|}
\hline 229 & 1644 & 593 & 430 & 2039 & 1835 \\
155042
\end{tabular}

\begin{tabular}{|l|l|l|l|l|l|}
\hline 229 & 1646 & 593 & 430 & 2043 & 1838 \\
\hline
\end{tabular}

\begin{tabular}{r|r|r|r|r|r|}
24 & 24 & 24 & 11 & 21 & 25 \\
\hline
\end{tabular}

27

\begin{tabular}{|r|r|l|l|}
\hline & 155790 & & \\
\hline 105 & & & \\
\hline & 108 & & \\
\hline
\end{tabular}


Table 4-1 Species found in fewer than 9 localities

\begin{tabular}{|c|c|}
\hline Species & Locality and Number ( ${ }^{*}=$ Count Period) \\
\hline Cackling Goose & Gardiner Dam (1) \\
\hline American Wigeon & Coronach (1) \\
\hline Blue-winged Teal & Coronach (1) \\
\hline Northern Pintail & Coronach (4), Yorkton (2) \\
\hline Green-winged Teal & Yorkton (2) \\
\hline Canvasback & Round Lake (2), Saskatoon $\left(1^{*}\right)$ \\
\hline Redhead & $\begin{array}{l}\text { Crooked Lake (1), Fort Qu'Appelle (2), Qu'Appelle Dam (8), } \\
\text { Squaw Rapids (1) }\end{array}$ \\
\hline Ring-necked Duck & Crooked Lake (1), Gardiner Dam(1) \\
\hline Lesser Scaup & $\begin{array}{l}\text { Crooked Lake (2), Estevan }\left(3^{*}\right) \text {, Gardiner Dam (24), Round Lake (2), } \\
\text { Saskatoon (1) }\end{array}$ \\
\hline Bufflehead & Coronach (1), Squaw Rapids (1) \\
\hline Common Merganser & $\begin{array}{l}\text { Estevan }\left(1^{*}\right) \text {, Gardiner Dam }(97) \text {, Indian Head (1), } \\
\text { Qu'Appelle Dam (31), Saskatoon }\left(1^{\star}\right) \text {, Spinney Hill (1), } \\
\text { Squaw Rapids (8) }\end{array}$ \\
\hline Spruce Grouse & LaRonge North (1), Meadow Lake (1), Turtle Lake (3) \\
\hline Wild Turkey & Broadview (9), Fort Walsh (1), Leader North (7) \\
\hline Double-crested Cormorant & Gardiner Dam (1) \\
\hline Turkey Vulture & Indian Head (3) \\
\hline Sharp-shinned Hawk & Archerwill (1), Craven $\left(1^{\star}\right)$ \\
\hline Cooper's Hawk & Clark's Crossing (1), Saskatoon (1) \\
\hline Red-tailed Hawk & Cypress Hills PP (1), Kamsack (2) \\
\hline American Kestrel & Indian Head (1) \\
\hline Peregrine Falcon & Gardiner Dam (1), Sask. Landing PP (1) \\
\hline Prairie Falcon & $\begin{array}{l}\text { Cabri (1), Estuary North (1), Govenlock (1), Kindersley North }\left(1^{*}\right) \text {, } \\
\text { Kyle }\left(1^{*}\right) \text {, Qu'Appelle Dam (1), Regina (1) }\end{array}$ \\
\hline American Coot & Estevan $\left(11^{\star}\right)$ \\
\hline Sandhill Crane & Cabri $\left(2^{*}\right)$ \\
\hline Wilson's Snipe & $\begin{array}{l}\text { Clark's Crossing (1), Fort Walsh (1), Qu'Appelle Dam (1), } \\
\text { Sask. Landing PP (1) }\end{array}$ \\
\hline Herring Gull & Gardiner Dam (2), Qu'Appelle Dam(1) \\
\hline Glaucous Gull & Gardiner Dam (4), Qu'Appelle Dam(1) \\
\hline Eurasian Collared-Dove & Moose Jaw (18), Morse (1), Swift Current $\left(3^{*}\right)$, Weyburn (2) \\
\hline Mourning Dove & Weyburn (1) \\
\hline Barred Owl & Endeavour (1), Mayview $\left(1^{*}\right)$ \\
\hline Long-eared Owl & Gardiner Dam (1), Sask. Landing PP (1) \\
\hline Boreal Owl & Round Lake $\left(1^{*}\right)$ \\
\hline Northern Saw-whet Owl & $\begin{array}{l}\text { Crooked Lake (1), Eastend }\left(1^{\star}\right), \text { Endeavour (1), Indian Head (1), } \\
\text { Saskatoon }\left(1^{*}\right), \text { Togo }(1)\end{array}$ \\
\hline Am. Three-toed Woodpecker & $\begin{array}{l}\text { Duck Lake (2), Emma Lake }\left(1^{\star}\right) \text {, Regina (1), Sask. R. Forks (1), } \\
\text { Spinney Hill (1), Squaw Rapids (1) }\end{array}$ \\
\hline Black-backed Woodpecker & $\begin{array}{l}\text { Emma Lake }\left(1^{*}\right) \text {, Kinloch }(1) \text {, Nisbet Forest NW }\left(1^{*}\right), \\
\text { Prince Albert NP }(2) \text {, Spruce Home SW }\left(1^{*}\right) \text {, Squaw Rapids (3) }\end{array}$ \\
\hline Loggerhead Shrike & Fort Walsh (1) \\
\hline American Crow & $\begin{array}{l}\text { Leader North (1), Regina (1), Saskatoon (3), Turtleford (1), } \\
\text { Weyburn (1), Yorkton (19) }\end{array}$ \\
\hline Brown Creeper & $\begin{array}{l}\text { Bangor (6), Cypress Hills PP (11), Estevan (1), Fort Walsh (1), } \\
\text { Regina (3), Sask. Landing PP (4), Saskatoon (2), Swift Current (7) }\end{array}$ \\
\hline American Dipper & Eastend (1) \\
\hline Townsend's Solitaire & $\begin{array}{l}\text { Cypress Hills PP (1), Eastend (1), Raymore (1), Saskatoon (2), } \\
\text { Swift Current (1) }\end{array}$ \\
\hline American Robin & $\begin{array}{l}\text { Clark's Crossing (1), Fort Qu'Appelle }\left(1^{*}\right) \text {, Indian Head (2), } \\
\text { Pike Lake (3), Saltcoats (1) }\end{array}$ \\
\hline
\end{tabular}


Table 4-2 Species found in fewer than 9 localities

\begin{tabular}{|c|c|}
\hline Species & Locality and Number $\left({ }^{*}=\right.$ Count Period $)$ \\
\hline Varied Thrush & $\begin{array}{l}\text { Little Manitou Lake (1), Moose Jaw (1), Pike Lake (1), Regina (1), } \\
\text { Swift Current (1) }\end{array}$ \\
\hline Spotted Towhee & Cypress Hills PP (1) \\
\hline American Tree Sparrow & $\begin{array}{l}\text { Bromhead (2), Cypress Hills PP (1), Eastend (5), Fort Walsh (36), } \\
\text { Last Mountain Lake }\left(1^{*}\right) \text {, Qu'Appelle Dam (4), Sask. Landing PP (8), } \\
\text { Sask. R. Forks (1) }\end{array}$ \\
\hline Chipping Sparrow & Armit (2), Indian Head (1) \\
\hline Vesper Sparrow & Yorkton (2) \\
\hline Fox Sparrow & Hudson Bay West (3) \\
\hline Song Sparrow & Fort Walsh (1) \\
\hline White-throated Sparrow & Armit (10), Kindersley North (1), Saskatoon (2), Swift Current (3) \\
\hline Harris's Sparrow & Fort Walsh (1), Raymore $\left(1^{*}\right)$, Skull Creek (25), Yorkton (6) \\
\hline White-crowned Sparrow & Fort Walsh (2), Pike Lake (1), Regina (1), Saskatoon $\left(1^{*}\right)$ \\
\hline McCown's Longspur & Coronach (10) \\
\hline Lapland Longspur & $\begin{array}{l}\text { Bromhead (27), Coronach (50), Govenlock (2), Grasslands NP (45), } \\
\text { Shamrock (60), Spinney Hill (3) }\end{array}$ \\
\hline Red-winged Blackbird & Fort Walsh(4), Regina (7) \\
\hline Western Meadowlark & Skull Creek (1) \\
\hline Rusty Blackbird & Estevan (2), Fort Qu'Appelle $\left(6^{\star}\right)$, Raymore $\left(1^{\star}\right)$, Swift Current (1) \\
\hline Brewer's Blackbird & Hudson Bay West (1) \\
\hline Common Grackle & Eastend (1), Hudson Bay West (1), Regina (2), Saskatoon (1) \\
\hline Gray-crowned Rosy-Finch & Eastend (1), Indian Head (1), Pike Lake (1), Spruce Home SW (2) \\
\hline Purple Finch & $\begin{array}{l}\text { Armit (4), Codette Lake (42), Fort Qu'Appelle (20), Fort Walsh (1), } \\
\text { Indian Head (8), Prince Albert (1), Rouleau (13) }\end{array}$ \\
\hline Red Crossbill & $\begin{array}{l}\text { Cypress Hills PP (8), Fort Qu'Appelle }\left(1^{*}\right) \text {, Indian Head (2), } \\
\text { Regina (10), Saskatoon (10) }\end{array}$ \\
\hline White-winged Crossbill & $\begin{array}{l}\text { Biggar }\left(2^{\star}\right) \text {, Broadview (4), Cypress Hills PP (2), Eastend (30), } \\
\text { Indian Head (3), Kilwinning (5), Raymore (2), Regina (35), } \\
\text { Saskatoon (30) }\end{array}$ \\
\hline American Goldfinch & $\begin{array}{l}\text { Craven (10), Fort Qu'Appelle }\left(1^{\star}\right) \text {, Indian Head (2), Kenosee Lake (1), } \\
\text { Regina (2), Saskatoon (1) }\end{array}$ \\
\hline
\end{tabular}

Table 5 Birds not identified to species

\begin{tabular}{|l|l|}
\hline Category & Locality and Number $~_{*}^{*}=$ Count Period) \\
\hline Swan sp. & Gardiner Dam (2) \\
\hline Duck sp. (Aythya) & Saskatoon (1) \\
\hline Eagle sp. & Kyle $(1)$ \\
\hline Buteo sp. & Saltcoats $\left(1^{\star}\right)$, Saskatoon $(1)$, Shamrock $(1)$ \\
\hline Large Falcon sp. & Coronach (1), Matador (1) \\
\hline Woodpecker sp. & Candle Lake (1) \\
\hline Sparrow sp. & Birch Hills (1), Grasslands NP (4), Prince Albert (3) \\
\hline Crossbill sp. & Duck Lake (2) \\
\hline
\end{tabular}


Table 6. New (in bold and italics) and tying high counts for individual species 2004. Count period results are in brackets.

\begin{tabular}{|c|c|c|c|c|}
\hline LOCATION & $\begin{array}{r}2004 \\
\text { COUNT }\end{array}$ & SPECIES & $\begin{array}{r}\text { PREVIOUS } \\
\text { HIGH }\end{array}$ & LOCATION, YEAR \\
\hline Gardiner Dam & 1 & Cackling Goose & New & \\
\hline Gardiner Dam & 24 & Lesser Scaup & 15 & Regina 1971 \\
\hline Gardiner Dam & 1 & $\begin{array}{l}\text { Double-crested } \\
\text { Cormorant } \\
\end{array}$ & 1 & $\begin{array}{l}\text { On previous counts north to } \\
\text { Squaw Rapids }\end{array}$ \\
\hline $\begin{array}{l}\text { Saskatchewan } \\
\text { Landing P.P. }\end{array}$ & 1 & Peregrine Falcon & 1 & $\begin{array}{l}\text { On previous counts north to } \\
\text { Squaw Rapids-Carrot River }\end{array}$ \\
\hline Cabri & (2) & Sandhill Crane & 1 & Yorkton 2003 \\
\hline Gardiner Dam & 4 & Glaucous Gull & 4 & $\begin{array}{l}\text { Gardiner Dam 1987, 1988, } \\
1990\end{array}$ \\
\hline Moose Jaw & 18 & $\begin{array}{l}\text { Eurasian Collared- } \\
\text { Dove }\end{array}$ & 8 & Moose Jaw 2001 \\
\hline Kyle & 28 & Great Horned OwI & 21 & Saskatoon 1987 \\
\hline $\begin{array}{l}\text { Nisbet Forest } \\
\text { West }\end{array}$ & 4 & Northern Hawk Owl & 4 & $\begin{array}{l}\text { Armit 1992, Crooked River } \\
1992\end{array}$ \\
\hline Kyle & 37 & Short-eared Owl & 21 & Saskatoon 1987 \\
\hline Round Lake & (1) & Boreal Owl & 1 & $\begin{array}{l}\text { On previous counts south } \\
\text { to Indian Head }\end{array}$ \\
\hline $\begin{array}{l}6 \text { counts (see } \\
\text { Table 4.) } \\
\end{array}$ & 1 & $\begin{array}{l}\text { Northern Saw-whet } \\
\text { Owl }\end{array}$ & 1 & $\begin{array}{l}\text { On previous counts north to } \\
\text { Maidstone Bridge }\end{array}$ \\
\hline Fort Walsh & 1 & Loggerhead Shrike & 1 & $\begin{array}{l}\text { Fort Walsh 1981, Regina } \\
\text { 1982, Fife Lake (1994) } \\
\end{array}$ \\
\hline Snowden & 360 & Common Raven & 279 & Nipawin 1993 \\
\hline $\begin{array}{l}\text { Cypress Hills } \\
P . P \text {. }\end{array}$ & 11 & Brown Creeper & 10 & Fort Walsh 2001 \\
\hline Eastend & 1 & American Dipper & New & \\
\hline $\begin{array}{l}\text { Cypress Hills } \\
\text { P.P. }\end{array}$ & 1 & Spotted Towhee & 1 & $\begin{array}{l}\text { On previous counts north to } \\
\text { Turtle Lake and Saskatoon }\end{array}$ \\
\hline Hudson Bay & 3 & Fox Sparrow & 1 & $\begin{array}{l}\text { On previous counts north } \\
\text { to Turtle Lake and } \\
\text { Saskatoon }\end{array}$ \\
\hline Yorkton & 2 & Vesper Sparrow & 2 & Swift Current 1993 \\
\hline Armit & 10 & $\begin{array}{l}\text { White-throated } \\
\text { Sparrow }\end{array}$ & 7 & Swift Current 1996 \\
\hline Skull Creek & 25 & Harris's Sparrow & 15 & Skull Creek 1992 \\
\hline $\begin{array}{l}\text { Fort } \\
\text { Qu'Appelle }\end{array}$ & 2 & $\begin{array}{l}\text { White-crowned } \\
\text { Sparrow }\end{array}$ & 2 & $\begin{array}{l}\text { Craven 1999, Swift Current } \\
2003\end{array}$ \\
\hline Coronach & 10 & McCown's Longspur & 1 & Govenlock 1982 \\
\hline
\end{tabular}


Table 7. Population changes in numbers of selected species in $2004^{*}$ compared to 2003 and the average for 5 years (1999-2003), based on the number of birds per party hour. A minus sign indicates a decrease from previous years.

\begin{tabular}{|c|c|c|c|c|c|}
\hline Species & $\begin{array}{l}\text { O } \\
8 \\
0 \\
\varepsilon \\
0 \\
0 \\
0 \\
0 \\
\frac{1}{2} \\
\frac{1}{N} \\
\frac{c}{0} \\
0\end{array}$ & 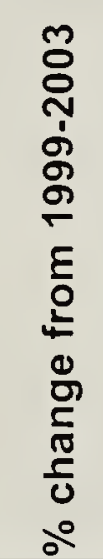 & & 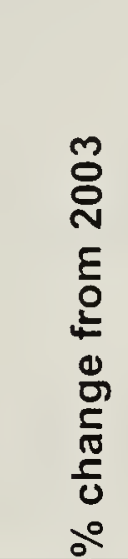 & 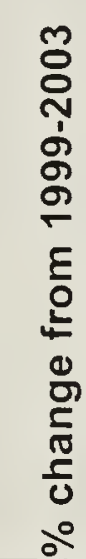 \\
\hline Canada Goose & 222 & 32 & Black-billed Magpie & -18 & -24 \\
\hline Mallard & 895 & 50 & American Crow & -27 & 13 \\
\hline Common Goldeneye & -27 & -23 & Common Raven & -9 & -1 \\
\hline Common Merganser & -18 & -24 & Horned Lark & 1186 & -13 \\
\hline Gray Partridge & -55 & 3 & Black-capped Chickadee & -6 & -3 \\
\hline Ring-necked Pheasant & -68 & -33 & Boreal Chickadee & -60 & -65 \\
\hline Ruffed Grouse & -61 & -46 & Red-breasted Nuthatch & -43 & -34 \\
\hline Sharp-tailed Grouse & -70 & -32 & White-breasted Nuthatch & 2 & -19 \\
\hline Bald Eagle & -16 & -7 & Brown Creeper & 60 & 146 \\
\hline Northern Goshawk & 14 & 50 & Golden-crowned Kinglet & 42 & -13 \\
\hline Rough-legged Hawk & -17 & 2 & American Robin & -88 & -95 \\
\hline Golden Eagle & -7 & 10 & European Starling & -49 & -50 \\
\hline Merlin & 34 & 69 & Bohemian Waxwing & 33 & 3 \\
\hline Prairie Falcon & 15 & -39 & Cedar Waxwing & 145 & 86 \\
\hline Rock Pigeon & -19 & -17 & American Tree Sparrow & 173 & -11 \\
\hline Great Horned Owl & 35 & 44 & Dark-eyed Junco & -61 & -31 \\
\hline Snowy Owl & 72 & 10 & Lapland Longspur & 497 & -84 \\
\hline Great Gray Owl & 152 & -19 & Snow Bunting & 168 & 35 \\
\hline Short-eared Owl & 2439 & 310 & Pine Grosbeak & -13 & 38 \\
\hline Downy Woodpecker & -15 & -2 & Purple Finch & 42 & -9 \\
\hline Hairy Woodpecker & -13 & 2 & House Finch & 3 & 60 \\
\hline Black-backed Woodpecker & -70 & -45 & White-winged Crossbill & -87 & -61 \\
\hline Northern Flicker & -30 & -24 & Common Redpoll & -35 & -11 \\
\hline Pileated Woodpecker & -44 & -5 & Hoary Redpoll & -49 & -28 \\
\hline Northern Shrike & 352 & 142 & Pine Siskin & 11 & 4 \\
\hline Gray Jay & -60 & -52 & Evening Grosbeak & -7 & -11 \\
\hline Blue Jay & 36 & 16 & House Sparrow & -25 & 1 \\
\hline
\end{tabular}

*As tremendous changes can occur in small sample sizes only those species recorded on an annual average of six or more counts are included. 